\title{
A Solvable Continuous Time Dynamic Principal-Agent Model
}

\author{
Noah Williams* \\ Department of Economics, University of Wisconsin - Madison \\ E-mail: nmwilliams@wisc.edu \\ Revised June 27, 2015
}

\begin{abstract}
I study the provision of incentives in a continuous time dynamic moral hazard model with hidden actions and hidden states. I consider a principal-agent model with linear production and exponential utility, whose explicit solution allows me to show how allocations are distorted for incentive reasons, and how access to hidden savings further alters allocations. I solve the model by applying a stochastic maximum principle, where the co-state variables from the agent's optimization problem become state variables for the principal's problem of choosing an optimal contract. I show that the main effect of moral hazard is a distortion on the effort margin, with a smaller effect on the intertemporal consumption allocation. Access to hidden savings shuts down the intertemporal distortions and increases the effort distortion. I also show how the optimal contracts can be implemented via a constant equity share, a constant flow payment, and a constant tax on savings.
\end{abstract}

\section{INTRODUCTION}

There are many economic environments where private information is a crucial feature, and a key question is how to provide incentives in a dynamic setting. However the analysis of dynamic hidden information models rapidly becomes complex, even more so when some of the relevant state variables cannot be monitored. In this paper I illustrate how continuous time methods help to simplify the analysis of models with hidden actions and hidden states. I study a simple continuous time contracting model where optimal contracts can be solved explicitly in closed form. This allows me to illustrate the effects of the information frictions, and to derive a simple implementation of the contract.

In particular, I study a dynamic principal-agent model that is an extension of Holmstrom and Milgrom (1987). In the model the principal owns a production technology, and hires an agent whose effort increases output. The proceeds of the production increase the principal's assets, out of which he draws funds for the agent's payment and his own consumption. I make the particular functional form assumptions that the production technology and asset accumulation are linear with additive shocks, and that both the principal and agent have exponential preferences over consumption, and the agent has quadratic financial costs of

\footnotetext{
* I thank the Alessandro Pavan (the editor), the associate editor, and two anonymous referees for useful suggestions. This is a substantially revised version of Williams (2008). I thank Dilip Abreu, Jakša Cvitanić, Narayana Kocherlakota, Nicola Pavoni, Chris Phelan, Bernard Salanié, Yuliy Sannikov, Ivan Werning, and Xun Yu Zhou for helpful comments on earlier versions of the paper. Financial support from the National Science Foundation is gratefully acknowledged.
} 
effort. This exponential-linear structure is useful for simplifying the analysis and allowing for explicit solutions, but the approach and methods apply more broadly. ${ }^{1}$

In this environment I study three different information structures. When the principal has full information, he can monitor the agent's effort and consumption, and so only must ensure that the agent be willing to participate in the contract. This case serves as an efficient benchmark to compare with the private information models. I then turn to the hidden action case, a classic moral hazard model where the principal cannot observe the agent's effort and so cannot distinguish between low effort or adverse shocks to output. Thus the contract must provide incentives for the agent to put forth effort, but I continue to assume that the agent's consumption is observable. As in Williams (2011) and Cvitanic and Zhang (2012), I apply a stochastic maximum principle to derive my results. ${ }^{2}$ I rely on the formulation of Bismut (1973), and consider a change of variables as in Bismut (1978). In this environment, I show that the first-order approach to contracting is valid. That is, the first order conditions for the agent's effort choice facing a given contract completely characterize the incentive constraints. Thus I can fully characterize the set of implementable contracts, where the agent participates and carries out the principal's desired actions. Implementable contracts incorporate the agent's first-order conditions and are history-dependent in that they condition on the agent's promised utility under the contract. This form of history dependence is well-known and is analogous to many related results in the literature, starting with Abreu, Pearce, and Stacchetti (1986)-(1990) and Spear and Srivastrava (1987). It has also been known at least since Mirrlees (1999) (originally 1975), that the first-order approach to contracting is not generally valid, but it is in my model. ${ }^{3}$ Closely related continuous time models have been studied by Sannikov (2008) and Cvitanic and Zhang (2012), among others, and Sannikov (2013) provides an overview of the literature. My contribution is in showing how to explicitly solve for contracts in a fully dynamic environment, a natural state variable (the assets) and consumption payments occurring continuously throughout the contract. ${ }^{4}$

I then turn to the case in which the agent has access to hidden savings, and so his consumption and wealth cannot be monitored. This introduces a hidden state variable, which is a form of persistent private information. As in Williams (2011), when the agent has access to a hidden state, the history dependence in the contract is captured by an additional state variable which summarizes the "shadow value" of the state. In this environment with hidden savings, that shadow value of additional wealth is the agent's current marginal utility of consumption. Again I employ a first-order approach to contracting, which is similar to

\footnotetext{
${ }^{1}$ In addition to Holmstrom and Milgrom (1987), other papers making use of the exponential-linear structure in related models include Fudenberg, Holmstrom, and Milgrom (1990), Mitchell and Zhang (2010), Cvitanic and Zhang (2012), and Williams (2011). In an earlier version of this paper, Williams (2008), I considered more general specifications.

${ }^{2}$ I developed my approach independently of Cvitanic and Zhang (2012), and they cite Williams (2008), the earlier version of this paper. Williams (2011) builds on Williams (2008).

${ }^{3}$ Different conditions insuring the validity of the first-order approach in a static setting were given by Mirrlees (1999), Rogerson (1985), and Jewitt (1988).

${ }^{4}$ Holmstrom and Milgrom (1987), Schattler and Sung (1993), and Cvitanić, Wan, and Zhang (2009) consider only a single transfer from the principal to the agent, as do the explicitly solved examples in Cvitanic and Zhang (2012). Sannikov (2008) considers dynamic payments but has no state variables other than promised utility.
} 
the approach of Werning (2001), Abraham and Pavoni (2008), Farhi and Werning (2013), Kapicka (2013), and Pavan, Segal, and Toikka (2014) in discrete time dynamic moral hazard problems. ${ }^{5}$ Garrett and Pavan (2015) consider an alternative approach using variational techniques to deal directly with the full program and characterize the dynamics of average distortions under optimal contracts. Unlike the hidden action case and Williams (2011), with hidden savings I cannot provide useful conditions which guarantee the validity of the first-order approach ex-ante. ${ }^{6}$ Therefore I derive a candidate optimal contract using the agent's (necessary) optimality conditions, and then verify ex-post that the contract is in fact implementable. Most previous papers in the literature have done this numerically, but here I am able to do so analytically. ${ }^{7}$ Similar to Holmstrom and Milgrom (1987) the optimal contract is linear in my fully dynamic setting with exponential utility. However now the payment is linear in an endogenous object, the logarithm of the agent's promised utility under the contract.

After solving for the optimal contracts, I study some of their implications. I characterize how the informational frictions distort the labor/leisure margin (a "labor wedge") and the consumption/savings margin (an "intertemporal wedge"). ${ }^{8}$ Moral hazard in production leads directly to a wedge between the agent's marginal rate of substitution and the marginal product of labor. Moreover, the dynamic nature of the contracting problem leads to a wedge between the agent's intertemporal marginal rate of substitution and the marginal product of capital. If the principal can control the agent's consumption (or tax his savings), the contract will thus have an intertemporal wedge. However with hidden savings, the agent's consumption cannot be directly controlled by the principal. Thus there is no intertemporal wedge, but instead a larger labor wedge. Finally, I show how the contracts can be implemented with some rather simple instruments. If the principal provides the agent with a constant share of output (or equity share), a constant flow payment, and a constant tax on savings, then the contracts in all of the information structures can be implemented in a decentralized manner with the agent free to choose his own effort, savings, and consumption.

\section{THE MODEL}

I consider a model in which a principal hires an agent to manage a risky project, with the agent's effort choice affecting the output from the project. Holmstrom and Milgrom (1987) study a related model where consumption and payments occur only at the end of the contracting period and output is i.i.d. I extend their environment in several ways. First, I include intermediate consumption by both the principal and agent. In addition, I study a

\footnotetext{
${ }^{5}$ Also see Allen (1985), Cole and Kocherlakota (2001), Kocherlakota (2004a), Doepke and Townsend (2006), Mitchell and Zhang (2010), and Edmans, Gabaix, Sadzik, and Sannikov (2012) for some alternative formulations with hidden savings.

${ }^{6}$ In Williams (2008) I provided some sufficient conditions which proved stringent and difficult to verify. The model in Williams (2011) has a different structure, which allows for weaker sufficient conditions.

${ }^{7}$ Mitchell and Zhang (2010) and Edmans, Gabaix, Sadzik, and Sannikov (2012) show that their contracts are incentive compatible with hidden savings via different methods.

${ }^{8}$ See Chari, Kehoe, and McGrattan (2007) on the role of the labor and intertemporal (or investment) wedges in fluctuations. Kocherlakota (2004b) shows how intertemporal wedges result form dynamic moral hazard problems.
} 
system with a natural state variable, where effort adds to the principal's stock of assets but consumption is drawn from it, which introduces a source of (observable) persistence. ${ }^{9}$ I also consider an extension with hidden savings, where the agent can save or borrow in a risk-free asset with a constant rate of return. In this case, my results are related to the discrete time model of Fudenberg, Holmstrom, and Milgrom (1990), who show that the hidden savings problem is greatly simplified with exponential preferences. Much of the complications of hidden savings comes through the interaction of wealth effects and incentive constraints, which exponential utility does away with. However my results are not quite as simple as theirs, as in my model the principal is risk averse, and asset stocks are persistent. ${ }^{10}$ Nonetheless, hidden savings affects the contract in a fairly simple way.

\subsection{The model}

The environment is a continuous time stochastic setting, with an underlying probability space $(\Omega, \mathcal{F}, P)$, on which is defined a standard Brownian motion $W$. Later I am more explicit about the probability space that I work with, focusing on the induced distributions over outcomes on a finite horizon. Information is represented by a filtration $\left\{\mathcal{F}_{t}\right\}$, which is generated by the Brownian motion $W_{t}$ (suitably augmented). I consider a finite horizon $[0, T]$ which may be arbitrarily long, and below I let $T \rightarrow \infty$.

At date zero, the principal contracts with the agent to manage a production process, whose cumulative proceeds $Y_{t}$ evolve on $[0, T]$ as follows:

$$
d Y_{t}=B e_{t} d t+\sigma d W_{t}
$$

here $e_{t} \in A \subset \mathbb{R}$ is the agent's effort choice, $B$ represents the productivity of effort, and there is an additive shock (due to the Brownian increment) to the output. The proceeds of the production add to the principal's assets $y_{t}$, which earn a risk free return $r$, and out of which he pays the agent $s_{t} \in S \subset \mathbb{R}$ and withdraws his own consumption (or dividend) $d_{t} \in \mathbb{R}$. Thus the principal's assets evolve as:

$$
d y_{t}=\left(r y_{t}+B e_{t}-s_{t}-d_{t}\right) d t+\sigma d W_{t}
$$

As is common when working with exponential-linear models, for tractability I do not restrict $y_{t}, s_{t}$, or $d_{t}$ to be nonnegative, thus their interpretations of may be a bit strained when they take on negative values. I sometimes refer to $y_{t}$ as "output," as from the principal's vantage point it captures the same information as $Y_{t}$. In addition, the agent has his own wealth $m_{t}$, out of which he consumes $c_{t}$ :

$$
d m_{t}=\left(r m_{t}+s_{t}-c_{t}\right) d t
$$

\footnotetext{
${ }^{9}$ Holmstrom and Milgrom (1987) also consider time multiplicatively separable preferences, while I use time additively separable ones.

${ }^{10}$ Fudenberg, Holmstrom, and Milgrom (1990) show that there are no gains to long term contracting, and that an optimal contract is completely independent of history. The first result relies on the risk neutrality of the principal, while the second relies on technology being history independent as well. Neither condition holds in my model, and I find that the optimal contract is history dependent and that hidden savings alter the contract.
} 
Thus the agent earns the same rate of return $r$ on his savings, gets income flows due to his payment $s_{t}$, and draws down his assets to consume. At the terminal date $T$, the principal makes a final payment $s_{T}$ and the agent chooses consumption based on this payment and his terminal wealth $m_{T}$. I provide more detail on the terminal date below.

I consider three different information structures: full information, hidden actions, and hidden savings. Full information is self-explanatory, while under hidden actions the principal observes the assets $y_{t}$ but cannot observe the agent's effort $e_{t}$ or the shocks $W_{t}$. Thus the principal faces a classic moral hazard problem, where he cannot distinguish low effort from a negative shock. He sets a target $\hat{e}_{t}$ but cannot directly verify if the agent were to deviate, and thus must structure the payment $\left\{s_{t}\right\}$ to provide incentives for the agent to meet his effort target. Under both full information and hidden actions, the principal can observe the agent's wealth $m_{t}$ and so equivalently can observe consumption $c_{t}$. In these cases the agent's saving is redundant, as only total assets $y_{t}+m_{t}$ matter for determining the allocation. As in Cole and Kocherlakota (2001), without loss of generality I suppose that the principal does all the saving, and thus sets $m_{t} \equiv 0$ and so $c_{t}=s_{t} .{ }^{11}$ The final information structure is hidden savings, where the principal observes the agent's initial wealth $m_{0}$ but cannot monitor the agent's wealth $m_{t}$ or consumption $c_{t}$ for $t>0$. In this case, in addition to the target effort $\hat{e}_{t}$, the principal sets targets $\hat{m}_{t} \equiv 0$ and $\hat{c}_{t}=s_{t}$ which are not verifiable. Thus the payments $\left\{s_{t}\right\}$ must provide incentives to put forth effort and not to save (or borrow).

As discussed above, I focus on exponential preferences for both the principal and the agent, which allows me to obtain explicit solutions for the optimal contracts. The same general methodology for finding optimal contracts applies more broadly, but typically requires numerical solutions. The agent discounts at rate $\rho$, has a flow utility $u(c, e)=$ $-\exp \left(-\lambda\left(c-\frac{e^{2}}{2}\right)\right)$ over consumption and effort and a terminal utility $v$ over the terminal payment and final wealth (which I discuss more below):

$$
V(\bar{e}, \bar{c})=E_{0}\left[\int_{0}^{T} e^{-\rho t} u\left(c_{t}, e_{t}\right) d t+e^{-\rho T} v\left(s_{T}, m_{T}\right)\right]
$$

Here $V$ denotes the expected utility over the processes $(\bar{e}, \bar{c})$. Similarly, the principal discounts at the same rate $\rho$, has a flow utility $U(d)=-\exp (-\lambda d)$ over his own consumption and has terminal utility $L$ over the terminal payment:

$$
E_{0}\left[\int_{0}^{T} e^{-\rho t} U\left(d_{t}\right) d t+e^{-\rho T} L\left(y_{T}, s_{T}\right)\right] .
$$

The assumption of common risk aversion simplifies explicit analytic solutions, but could be relaxed without much complication. ${ }^{12}$

\subsection{The terminal date}

\footnotetext{
${ }^{11}$ This relies on risk being additive and hence independent of $y_{t}$. Otherwise varying $m_{t}$ may affect the risk of the total asset stock $y_{t}+m_{t}$, and the principal would face a portfolio problem.

${ }^{12}$ With differing risk aversion, the same general form of the solution holds, with slightly more complex expressions and exponents depending on the ratio of the risk aversion parameters.
} 
In order to find explicit solutions and to extend easily from a finite to an infinite horizon, it is useful to make particular assumptions about what happens at the terminal date. In particular, I assume that at the terminal date $T$, the principal provides the agent with an amount $s_{T}$, retaining $y_{T}-s_{T}$ for himself. Then from date $T$ on, no more production takes place, and both the principal and the agent live off their assets for the infinite future, earning the same constant rate of return $r$. That is, both solve consumption-savings problems of the form:

$$
V_{T}\left(a_{0}\right)=\max _{\left\{b_{t}\right\}}-\int_{0}^{\infty} \exp \left(-\rho t-\lambda b_{t}\right) d t
$$

with a generic level of assets $a_{0}$ given and:

$$
d a_{t}=\left(r a_{t}-b_{t}\right) d t
$$

For the agent $b_{t}=c_{t}$ and $a_{0}=s_{T}+m_{T}$, while for the principal $b_{t}=d_{t}$ and $a_{0}=y_{T}-s_{T}$.

The Hamilton-Jacobi-Bellman equation for the consumption-savings problem (5) is:

$$
\rho V_{T}(a)=\max _{b}\left\{-\exp (-\lambda b)+V_{T}^{\prime}(a)[r a-b]\right\} .
$$

One can easily verify that the solution and optimal policy are:

$$
V_{T}(a)=-\exp \left(\frac{\rho-r}{r}-\lambda r a\right), \quad b(a)=\frac{r-\rho}{\lambda r}+r a .
$$

Thus I set the terminal utility functions for the agent and the principal to:

$$
v\left(s_{T}, m_{T}\right)=V_{T}\left(s_{T}+m_{T}\right), \quad L\left(y_{T}, s_{T}\right)=V_{T}\left(y_{T}-s_{T}\right) .
$$

\subsection{Contracts and implementability}

I now more formally define contracts and what I mean for a contract to be implementable (or incentive compatible). Let $C$ be the space of continuous functions mapping $[0, T]$ into $\mathbb{R}$. I adopt the convention of letting a bar over a variable indicate an entire time path on $[0, T]$. The time path of output $\bar{y}=\left\{y_{t}: t \in[0, T]\right\}$ is then a (random) element of $C$, which defines the principal's observation path under hidden actions or hidden savings. I define the filtration $\left\{\mathcal{Y}_{t}\right\}$ to be the completion of the $\sigma$-algebra generated by $y_{t}$ at each date. A contract specifies a set of recommended actions $\left(\hat{e}_{t}, \hat{c}_{t}\right) \in A$ and a corresponding payment $s_{t} \in S$ for all $t$ as a function of the relevant history. For the hidden savings case, these recommended actions truly are recommendations, while under hidden actions $\hat{c}_{t}=c_{t}=s_{t}$, and under full information $\hat{c}_{t}=c_{t}=s_{t}$ and $\hat{e}_{t}=e_{t}$. The set of admissible contracts $\mathcal{S}$ is the set of $\mathcal{Y}_{t}$-predictable functions $(s, \hat{e}, \hat{c}):[0, T] \times C \rightarrow S \times A .{ }^{13}$ Thus the contract specifies a payment and recommendations at date $t$ that depend on the whole past history of the observations of the state up to that date (but not on the future). Then facing a given

\footnotetext{
${ }^{13}$ See Elliott (1982) for a definition of predictability. Any left-continuous, adapted process is predictable.
} 
contract, the agent potentially makes his own choices of effort and consumption. Under full information, the agent has no choices to make, while under hidden actions he chooses effort, and under hidden savings effort and consumption. Thus the set of admissible controls $\mathcal{A}$ for the agent are those $\mathcal{F}_{t}$-predictable functions $(\bar{e}, \bar{c}):[0, T] \times C \rightarrow A$. To apply maximum principle results from the literature, I assume that the set $A$ can be written as the countable union of compact sets. A contract $(s, \hat{e}, \hat{c})$ is called implementable if the agent agrees to the contract at date zero and chooses the recommended actions: $(\hat{e}, \hat{c})=(\bar{e}, \bar{c})$. Under full information, all contracts that ensure participation are implementable, while under hidden actions $\hat{c}=\bar{c}$, so implementable contracts are those with $\hat{e}=\bar{e}$.

\subsection{A change of variables}

Under hidden actions or hidden savings, I derive the incentive compatibility conditions by considering the decision problem of an agent facing a given contract. In general the payment at any date $t$ is $s_{t}=s(t, \bar{y})$, which is a function of the entire past history. This general history dependence means that we cannot use a direct approach to the agent's problem, as a function - the entire past history $\bar{y}$ - would be a state variable.

As in Bismut (1978), I make the problem tractable by taking the key state variable to be the density of the output process rather than the output process itself. Cvitanic and Zhang (2012) follow a similar approach. In particular, let $W_{t}^{0}$ be a Wiener process on $C$, which can be interpreted as the distribution of output resulting from an effort policy which makes output a martingale. Different effort choices by the agent change the distribution of output. Thus the agent's effort choice is a choice of a probability measure over output, and I take the relative density $\Gamma_{t}$ for this change of measure as the key state variable. Details of the change of measure are given in Appendix A.1, where I show that the density evolves as:

$$
d \Gamma_{t}=\Gamma_{t} \sigma^{-1}\left(r y_{t}+B e_{t}-s(t, \bar{y})-d_{t}\right) d W_{t}^{0},
$$

with $\Gamma_{0}=1 .^{14}$

When there are hidden states, the covariation between the observable and unobservable states is also a key factor in the model. Thus is it also useful to take the density-weighted wealth $x_{t}=\Gamma_{t} m_{t}$ as the relevant unobservable state variable. Simple calculations from (3) and (7) show that its evolution is:

$$
d x_{t}=\Gamma_{t}\left(r x_{t} / \Gamma_{t}+s(t, \bar{y})-c_{t}\right) d t+x_{t} \sigma^{-1}\left(r y_{t}+B e_{t}-s(t, \bar{y})-d_{t}\right) d W_{t}^{0},
$$

with $x_{0}=m_{0}$.

By changing variables from $\left(y_{t}, m_{t}\right)$ to $\left(\Gamma_{t}, x_{t}\right)$ the state evolution is now a stochastic differential equation with random coefficients. Instead of the key states directly depending on their entire past history, the coefficients of the transformed state evolution depend on $\bar{y}$ which is a fixed, but random, element of the probability space. That is, when analyzing the agent's problem I do not take the state variable to be $y_{t}$, and analyze how the agent's

\footnotetext{
${ }^{14}$ Similar ideas are employed by Elliott (1982), Schattler and Sung (1993), and Sannikov (2008) who use a similar change of measure in their martingale methods. Their approach does not apply in the hidden state case however.
} 
choice off effort affects future values of $y_{t}$, which in turn persist into the future because of the state dependence. Instead, I fix an outcome path $\bar{y}$, and view the agent as choosing effort to affect the likelihood of observing that outcome. Changing variables in this way allows me to deal with the history, as the relevant state variable for the agent's is now the current density $\Gamma_{t}$ instead of the whole past history of output.

\section{THE FULL INFORMATION PROBLEM}

I start with the full information problem which provides a benchmark for the asymmetric information models considered below. The full information problem is also the easiest to analyze, as the principal can observe the agent's effort and consumption, and thus specify their values directly in a contract. (Recall that the principal also sets $m_{t} \equiv 0$.) The only constraint on the contract is that the principal must induce the agent to participate at date zero, after which both parties remain committed to the contract. I assume that the agent has an outside reservation utility level $V_{0}$, and thus the contract must satisfy the participation constraint $V(\bar{e}, \bar{c}) \geq V_{0}$.

As the participation constraint only holds at date zero, it would be straightforward to impose it via standard Lagrangian methods. However from the literature starting with Abreu, Pearce, and Stacchetti (1986)-(1990) and Spear and Srivastrava (1987), we know that in a dynamic moral hazard setting a contract should condition on the agent's promised utility. Thus it is natural that promised utility should appear in the private information cases studied below, so I introduce it here as a way of imposing the participation constraint. Thus I define the agent's promised utility $q_{t}$ as the expected discounted utility for remaining in the contract from date $t$ forward:

$$
q_{t}=E\left[\int_{t}^{T} e^{-\rho(s-t)} u\left(c_{s}, e_{s}\right) d s+e^{-\rho(T-t)} v\left(s_{T}, 0\right) \mid \mathcal{F}_{t}\right]
$$

Via the martingale representation theorem, its evolution can be written:

$$
d q_{t}=\left[\rho q_{t}-u\left(c_{t}, e_{t}\right)\right] d t+\gamma_{t} \sigma d W_{t}, \quad q_{T}=v\left(s_{T}, 0\right) .
$$

Thus promised utility follows a backward stochastic differential equations (BSDE), as it has a specified terminal conditions but an unknown initial value. ${ }^{15}$ Here $\gamma_{t}$ gives the sensitivity of the agent's promised utility to the fundamental shocks, and is the key for providing incentives under moral hazard. Under full information, the principal can freely choose $\gamma_{t}$ as part of the contract, as long as it implies a solution of (10) which is consistent with the participation constraint $q_{0} \geq V_{0}$.

Thus under full information, the principal's problem is to choose $\left(c_{t}, d_{t}, e_{t}, \gamma_{t}\right)$ for all $t$ and the terminal payment $s_{T}$ in order to maximize his own utility (4), subject the evolution of assets (2) and promised utility (10), and the participation constraint. I define $J(t, y, q)$ as

\footnotetext{
${ }^{15}$ In particular, these BSDEs depend on forward SDEs, as described in Ma and Yong (1999) and Cvitanic and Zhang (2012).
} 
the principal's value function, capturing his maximal expected discounted value as of date $t$ when the current assets are $y_{t}=y$ and the agent's utility promise is $q_{t}=q$. This value function satisfies the Hamilton-Jacobi-Bellman (HJB) equation for $0<t<T$ :

$$
\begin{gathered}
\rho J(t, y, q)-J_{t}(t, y, q)=\max _{c, d, e, \gamma}\left\{-\exp (-\lambda d)+J_{y}(t, y, q)[r y+B e-c-d]\right. \\
\left.+J_{q}(t, y, q)\left[\rho q+\exp \left(-\lambda\left(c-e^{2} / 2\right)\right)\right]+\frac{1}{2} J_{y y}(t, y, q) \sigma^{2}+J_{y q}(t, y, q) \gamma \sigma^{2}+\frac{1}{2} J_{q q}(t, y, q) \gamma^{2} \sigma^{2}\right\},
\end{gathered}
$$

with the terminal condition $J\left(T, y_{T}, q_{T}\right)=V_{T}\left(y_{T}-s_{T}\right)$ where $q_{T}=V_{T}\left(s_{T}\right)$. Suppressing arguments of functions, the first order conditions for $(d, c, e, \gamma)$ are then:

$$
\begin{aligned}
\lambda \exp (-\lambda d) & =J_{y} \\
\lambda \exp \left(-\lambda\left(c-e^{2} / 2\right)\right) & =-J_{y} / J_{q} \\
\lambda e \exp \left(-\lambda\left(c-e^{2} / 2\right)\right) & =-B J_{y} / J_{q} \\
\gamma & =-J_{y q} / J_{q q}
\end{aligned}
$$

Taking ratios of the conditions for $(c, e)$ gives $e=B$. Thus it is optimal for the principal to require the agent to supply a constant effort, equal to his productivity $B$. This is simply a manifestation of the standard efficiency condition equating the agent's marginal rate of substitution between effort and consumption (which here is equal to $e$ ) to the marginal productivity of effort (which here is equal to $B$ ).

Due to the exponential preferences and linear evolution, I can explicitly solve for the optimal contract. First, the terminal condition and the specification of terminal preferences (6) gives the following:

$$
s_{T}=-\frac{\log \left(-q_{T} / \eta\right)}{\lambda r}
$$

where $\eta=\exp \left(\frac{\rho-r}{r}\right) / r$. Thus we have:

$$
J\left(T, y_{T}, q_{T}\right)=V_{T}\left(y_{T}-s_{T}\right)=\frac{\eta^{2}}{q_{T}} \exp \left(-\lambda r y_{T}\right) .
$$

The solution for any date $t$ has a similar form, and can be written:

$$
J(t, y, q)=\frac{j_{0}(t)}{q} \exp (-\lambda r y)
$$

for some function $j_{0}(t)$. Substituting this into the HJB equation yields the ODE:

$$
j_{0}^{\prime}(t)=j_{0}(t)\left[r \log j_{0}(t)-\alpha\right]
$$

where:

$$
\alpha=2(r-\rho)-\frac{\lambda r B^{2}}{2}+\frac{\sigma^{2} \lambda^{2} r^{2}}{4}-2 r \log r
$$


and $j_{0}(T)=\eta^{2}$. The solution of this ODE is thus:

$$
j_{0}(t)=\exp (\alpha / r+[2 \log \eta-\alpha / r] \exp [-r(T-t)]) .
$$

The optimal policies are therefore:

$$
\begin{aligned}
e^{f i} & =B, \quad \gamma^{f i}(q)=-\frac{\lambda r q}{2}, \\
c^{f i}(q) & =\frac{B^{2}}{2}-\frac{\log r}{\lambda}-\frac{\log (-q)}{\lambda}, \\
d^{f i}(t, y, q) & =-\frac{\log \left(r j_{0}(t)\right)}{\lambda}+\frac{\log (-q)}{\lambda}+r y .
\end{aligned}
$$

Since both the principal and agent are risk averse, they share the production risk. The agent's optimal effort is constant and his consumption does not depend directly on output, but instead is linear in the log of the utility process (which however is a function of output). The principal's consumption is linear in the log of the agent's utility process and also linear in current output. The principal's consumption is the only time-dependent policy function, capturing the finite horizon nature of the problem.

To ease some comparisons, I now consider the infinite horizon limit of the contract. In the full information case, the infinite horizon problem could be solved directly. However my theoretical results on private information models only apply to finite horizon cases, so I study the limit of these solutions as $T \rightarrow \infty$. Here we have $\lim _{T \rightarrow \infty} j_{0}(t)=\exp (\alpha / r)$, and one can verify directly that the solution of the infinite horizon problem is $J(y, q)=$ $\exp (\alpha / r) / q \exp (-\lambda r y)$. In the infinite horizon limit, the state variables evolve as follows:

$$
\begin{aligned}
& d y_{t}=\left[\frac{2(r-\rho)}{r \lambda}+\frac{\sigma^{2} \lambda r}{4}\right] d t+\sigma d W_{t} \\
& d q_{t}=(\rho-r) q_{t} d t-\frac{\sigma \lambda r}{2} q_{t} d W_{t} .
\end{aligned}
$$

Thus output follows an arithmetic Brownian motion with constant drift, while the utility process follows a geometric Brownian motion. The expected growth rate of utility is constant and equal to the difference between the subjective discount rate $\rho$ and the rate of return $r$.

\section{THE HIDDEN ACTION CASE}

I now turn to the first model with private information: a dynamic moral hazard setting where the principal can observe the assets $y_{t}$ but cannot observe the agent's effort $e_{t}$ (or the shocks $W_{t}$ ). I continue to assume that the agent's consumption and wealth are observable, and thus set $c_{t}=s_{t}$ and $m_{t} \equiv 0$. Thus the principal must provide incentives for the agent to put forth the desired amount of effort. I solve this problem by first deriving the agent's optimality conditions facing a given history-dependent contract, then showing that the first order conditions from the agent's problem are sufficient to ensure implementability. Then I explicitly solve for the optimal contract. 


\subsection{The agent's problem}

The first step in designing an incentive compatible contract is to see what effort level the agent would choose when facing a given contract. However, as discussed in Section 2.4, the history dependence in the contract makes a change of variables useful, so I take the density process $(7)$ as the relevant state variable. With $c_{t}=s(t, \bar{y})$ we can write the agent's preferences as:

$$
\begin{aligned}
V(\bar{e}, \bar{s}) & =E_{\bar{e}}\left[\int_{0}^{T} e^{-\rho t} u\left(s(t, \bar{y}), e_{t}\right) d t+v\left(s_{T}, 0\right)\right] \\
& =E\left[\int_{0}^{T} \Gamma_{t} e^{-\rho t} u\left(s(t, \bar{y}), e_{t}\right) d t+\Gamma_{T} v\left(s_{T}, 0\right)\right]
\end{aligned}
$$

Here the first line uses the expectation with respect to the measure $P_{\bar{e}}$ over output induced by the effort policy $\bar{e}$, as discussed in Appendix A.1. The second line uses the density process defined above, which effectively averages over the state dependence which is inherited from the contract. The agent's problem is to solve:

$$
\sup _{\bar{e} \in \mathcal{A}} V(\bar{e}, \bar{s})
$$

subject to (7), given $\bar{s}$.

Under the change of variables, the agent's problem is one of control with random coefficients. As in Williams (2011), I apply a stochastic maximum principle due to Bismut (1973) to characterize the agent's optimality conditions. ${ }^{16}$ Similar to the deterministic Pontryagin maximum principle, the stochastic maximum principle defines a Hamiltonian, expresses optimality conditions as differentials of it, and derives "co-state" or adjoint variables. However since now the state variable is stochastic, the adjoint variable consists of a pair of processes, one which multiplies the drift of the state and another the diffusion. The pair of adjoint processes together solve a a BSDE. In particular, the Hamiltonian $\mathcal{H}$ for the agent's problem with state $\Gamma_{t}$ and adjoint $\left(q_{t}, \gamma_{t}\right)$ can be defined as:

$$
\mathcal{H}=\Gamma H(y, e, c, d, \gamma)=\Gamma[u(c, e)+\gamma(r y+B e-c-d)]
$$

Since there is no drift in $\Gamma$, the level of the adjoint $q$ does not enter. In addition, since $\Gamma_{t}>0$, we can define the conditions on the (reduced) Hamiltonian $H$ rather than $\mathcal{H}$.

As in the deterministic theory, optimal controls maximize the Hamiltonian, and the evolution of the adjoint (or co-state) variables is governed by differentials of the Hamiltonian. In particular, the drift of the co-state is $-\frac{\partial \mathcal{H}}{\partial \Gamma}$ and a term reflecting the discounting, and we

\footnotetext{
16 The basic maximum principle is further exposited in Bismut (1978), and more recent contributions are detailed in Yong and Zhou (1999). Cvitanic and Zhang (2012) use a similar approach.
} 
add a diffusion term:

$$
\begin{aligned}
d q_{t} & =\left[\rho q_{t}-u\left(c_{t}, e_{t}\right)-\gamma_{t}\left(r y_{t}+B e_{t}-c_{t}-d_{t}\right)\right] d t+\sigma \gamma_{t} d W_{t}^{0} \\
& =\left[\rho q_{t}-u\left(c_{t}, e_{t}\right)\right] d t+\sigma \gamma_{t} d W_{t}^{\bar{e}} \\
q_{T} & =v\left(y_{T}, 0\right) .
\end{aligned}
$$

Here the second line carries out the change of measure, and shows that the co-state associated with the density of the change of measure $\Gamma_{t}$ is the promised utility $q_{t}$ as in (10). In the full information case we introduced this variable for convenience, but here it arises as an element of the agent's optimality conditions, capturing the shadow value of the change in the likelihood of different output processes.

In the following, I say that a process $X_{t} \in L^{2}$ if $E \int_{0}^{T} X_{t}^{2} d t<\infty$. The first result gives the necessary conditions for optimality. The proofs of it and all results to follow are in Appendix A.2.

Proposition 4.1. Let $\left(e^{*}, \Gamma^{*}\right)$ be an optimal control-state pair. Then there exists an $\mathcal{F}_{t}$-adapted process $\left(q_{t}, \gamma_{t}\right)$ in $L^{2}$ that satisfies (12) with $e=e^{*}$. Moreover the optimal choice $e^{*}$ satisfies for almost every $t \in[0, T]$ almost surely:

$$
H\left(y_{t}, e_{t}^{*}, c_{t}, d_{t}, \gamma_{t}\right)=\max _{e \in A} H\left(y_{t}, e, c_{t}, d_{t}, \gamma_{t}\right)
$$

Thus if $A$ is convex an optimal control $e^{*}$ must satisfy for all $e \in A$, almost surely:

$$
H_{e}\left(y_{t}, e_{t}^{*}, c_{t}, d_{t}, \gamma_{t}\right)\left(e-e_{t}^{*}\right) \leq 0
$$

I stress that these are only necessary conditions for the agent's problem, and thus the set of implementable contracts may be smaller than that characterized by the first order conditions alone. However, I establish the validity of my first-order approach in the next section.

\subsection{Implementable contracts}

I focus on interior target effort policies $\hat{e}$, and build in the incentive constraints via the first order condition (14), which thus reduces to an equality at $\hat{e}$ :

$$
\gamma_{t} B=-u_{e}\left(c_{t}, \hat{e}_{t}\right)=\lambda e_{t} \exp \left(-\lambda\left(c_{t}-\hat{e}_{t}^{2} / 2\right)\right) .
$$

This equation can then define the target volatility $\hat{\gamma}_{t}$ implied by the consumption and target effort under the contract. Notice as well that $\hat{\gamma}_{t}>0$, so that promised utility increases with a positive shock (positive increment to the Brownian motion $W_{t}$ ).

I now characterize the set of implementable contracts. I define a contract as locally incentive compatible if it satisfies the first order condition (15). This condition ensures that if an agent faced a contract $(\hat{c}, \hat{e})$ with associated volatility $\hat{\gamma}$, the target effort $\hat{e}$ would in fact be his optimal response:

$$
H\left(y_{t}, \hat{e}_{t}, \hat{c}_{t}, d_{t}, \hat{\gamma}_{t}\right)=\max _{e \in A} H\left(y_{t}, e, \hat{c}_{t}, d_{t}, \hat{\gamma}_{t}\right)
$$


In the model, the Hamiltonian $H$ is concave in $e$, so the first order condition (15) is necessary and sufficient for this local optimality. In addition, I say that a contract satisfies promise-keeping if it implies a solution of (12). Not all contracts satisfy this condition, as they may not guarantee that the terminal condition is satisfied. A contract satisfies the participation constraint if this solution has $q_{0} \geq V_{0}$. The next result shows that these conditions completely characterize the set of implementable contracts.

Proposition 4.2. A contract $(\hat{c}, \hat{e}) \in \mathcal{S}$ is implementable in the hidden action case if and only if it: (i) satisfies the participation constraint, (ii) satisfies promise-keeping, and (iii) is locally incentive compatible.

Thus in the setting of this paper, the first order approach is valid and the global optimality conditions can be reduced to the local first order condition. ${ }^{17}$

\subsection{The optimal contract}

I now consider the principal's choice of a contract and show how to solve the problem explicitly. Defining the value function $J(t, y, q)$ as above, the principal's problem is to choose a contract that satisfies participation, promise-keeping, and incentive constraints. Relative to the full information constraint, the volatility variable $\gamma$ is no longer a free choice, but instead is pinned down via (15) as a function of consumption and effort, $\gamma(c, e)$. The principal's HJB equation for $0<t<T$ now becomes:

$$
\begin{gathered}
\rho J(t, y, q)-J_{t}(t, y, q)=\max _{c, d, e}\left\{-\exp (-\lambda d)+J_{y}(t, y, q)[r y+B e-c-d]+\right. \\
\left.J_{q}(t, y, q)\left[\rho q+\exp \left(-\lambda\left(c-e^{2} / 2\right)\right)\right]+\frac{1}{2} J_{y y}(t, y, q) \sigma^{2}+J_{y q}(t, y, q) \gamma(c, e) \sigma^{2}+\frac{1}{2} J_{q q}(t, y, q) \gamma(c, e)^{2} \sigma^{2}\right\},
\end{gathered}
$$

again with the terminal condition $J\left(T, y_{T}, q_{T}\right)=V_{T}\left(y_{T}-s_{T}\right)$ where $q_{T}=V_{T}\left(s_{T}\right)$. The first order conditions for $(d, c, e)$, using the form of $\gamma(c, e)$ from (15) and suppressing function arguments, are:

$$
\begin{aligned}
\lambda \exp (-\lambda d) & =J_{y}, \\
-J_{y}-J_{q} \lambda \exp \left(-\lambda\left(c-e^{2} / 2\right)\right)-J_{y q} \sigma^{2} \lambda \gamma(c, e)-J_{q q} \sigma^{2} \lambda \gamma(c, e)^{2} & =0, \\
J_{y} B+J_{q} \lambda e \exp \left(-\lambda\left(c-1 / 2 e^{2}\right)\right)+J_{y q} \sigma^{2} \frac{1+\lambda e^{2}}{e} \gamma(c, e)+J_{q q} \sigma^{2} \frac{1+\lambda e^{2}}{e} \gamma(c, e)^{2} & =0 .
\end{aligned}
$$

A special feature of the exponential-linear environment is that the value functions and the optimal policies have the same form under private information as full information, albeit with different constants. In particular, the value function is of the same form:

$$
J(t, y, q)=\frac{j_{1}(t)}{q} \exp (-\lambda r y)
$$

\footnotetext{
${ }^{17}$ In an earlier version of this paper, Williams (2008), I considered more general preferences $u$ and production technologies $f$. There I showed that the first order approach was valid if $u$ and $f$ were concave in $e$ and $u_{e}<0$ and $f_{e}>0$. These natural assumptions, with effort lowering utility but increasing output, are clearly satisfied here.
} 
for some function $j_{1}(t)$. The optimal policies are:

$$
\begin{aligned}
e^{h a} & =e^{*}, \quad \gamma^{h a}(q)=-\frac{\lambda e^{*} k q}{B}, \\
c^{h a}(q) & =\frac{\left(e^{*}\right)^{2}}{2}-\frac{\log k}{\lambda}-\frac{\log (-q)}{\lambda}, \\
d^{h a}(t, y, q) & =-\frac{\log \left(j_{1}(t) r\right)}{\lambda}+\frac{\log (-q)}{\lambda}+r y,
\end{aligned}
$$

where $\left(e^{*}, k\right)$ are constants. Using the value function and the fact that $u\left(c^{h a}(q), e^{*}\right)=k q$ in the first order conditions for $(e, c)$, we see that $e^{*}$ and $k$ must satisfy:

$$
\begin{aligned}
r-k+\sigma^{2} r \lambda^{2} e^{*} k / B-2 \sigma^{2} \lambda^{2}\left(e^{*}\right)^{2} k^{2} / B^{2} & =0, \\
-B r+\lambda e^{*} k-\sigma^{2} r \lambda\left(1+\lambda\left(e^{*}\right)^{2}\right) k / B-2 \sigma^{2} \lambda e^{*}\left(1+\lambda\left(e^{*}\right)^{2}\right) k^{2} / B^{2} & =0 .
\end{aligned}
$$

These can be solved to get $e^{*}(k)$ :

$$
e^{*}(k)=\frac{B^{3} r+\sigma^{2} \lambda B r k}{B^{2} r+2 \sigma^{2} \lambda k^{2}},
$$

then substituting this back into the first equation in (16) gives an implicit expression for $k$.

The function $j_{1}(t)$ is determined from the HJB equation after substituting in the optimal policies. The function satisfies an ODE of the same form as under full information:

$$
j_{1}^{\prime}(t)=j_{1}(t)\left[r \log j_{1}(t)-\beta\right]
$$

where:

$$
\beta=r+k-2 \rho-r\left[B \lambda e^{*}-\lambda\left(e^{*}\right)^{2} / 2+\log (r k)\right]+r \lambda^{2} \sigma^{2}\left(r-e^{*} k / B+\lambda\left(e^{*}\right)^{2} k^{2} / B^{2}\right)
$$

and $j_{1}(T)=\eta^{2}$. The solution of this ODE is thus:

$$
j_{1}(t)=\exp (\beta / r+[2 \log (\eta)-\beta / r] \exp [-r(T-t)]) .
$$

While the form of the policy functions is the same as in the full information case, the constants defining them differ. Solving for the values of the constants is a simple numerical task, but explicit analytic expressions are not available. For $\sigma^{2}=0$ the policies collapse to the full information solution with $e^{*}=B$ and $k=r$, which is natural as there is clearly no information asymmetry when there are no shocks to output. For small $\sigma^{2}$ we have $e^{*} \approx B$ and $k \approx r$, so $\gamma^{h a} \approx-\lambda r q=2 \gamma^{f i}$. In order to provide incentives, moral hazard requires that the agent's utility be more responsive (here twice as much) to incoming information than would be optimal under full information.

To gain some additional insight into the optimal contract, I expand $e^{*}$ and $k$ in $\sigma^{2}$ around zero. From (16) and (17) I have the following approximations:

$$
\begin{aligned}
e^{*} & =B-\sigma^{2} \frac{\lambda r}{B}+o\left(\sigma^{4}\right) \\
k & =r-\sigma^{2} \lambda^{2} r^{2}+o\left(\sigma^{4}\right) .
\end{aligned}
$$


In turn, substituting these approximations into $c^{h a}(q)$ gives:

$$
c^{h a}(q)=c^{f i}(q)+o\left(\sigma^{4}\right) .
$$

Thus the first order effects (in the shock variance) of the information frictions are a reduction in effort put forth, but no change in consumption. I show below that $k$ is the agent's effective rate of return under the contract, and can be implemented as an after-tax return on savings. Thus this return decreases with more volatility. Moreover, effort varies with the parameters in a simple way: a greater rate of return parameter $r$ or risk aversion parameter $\lambda$ or smaller productivity values $B$ lead to larger reductions in effort. Below I plot the exact solutions for a parameterized version of the model and show that the results are in accord with these approximations. Thus the information friction leads to a reduction in effort, but has little effect on consumption.

As discussed above, the theoretical results only apply to finite horizon cases, and there are important technical issues involved in carrying out the change of measure on an infinite horizon. But the limit of the finite horizon solution is well defined. Similar to the full information case, we have $\lim _{T \rightarrow \infty} j_{1}(t)=\exp (\beta / r)$. In the infinite horizon limit, the state variables evolve as follows:

$$
\begin{aligned}
& d y_{t}=\left[\frac{(r+k-2 \rho)}{r \lambda}+\sigma^{2} \lambda\left(\frac{r}{2}-\frac{e^{*} k}{B}+\frac{\left(e^{*}\right)^{2} k^{2}}{r B^{2}}\right)\right] d t+\sigma d W_{t} \\
& d q_{t}=(\rho-k) q_{t} d t-\frac{\sigma \lambda e^{*} k}{B} q_{t} d W_{t} .
\end{aligned}
$$

Thus output again follows an arithmetic Brownian motion with constant drift, while the utility process follows a geometric Brownian motion. Relative to full information, moral hazard increases the expected growth rate of promised utility, as (at least for small $\sigma^{2}$ ) $k<r$, and at the same time increases its volatility as $\gamma^{h a}>\gamma^{f i}$.

\section{THE HIDDEN SAVINGS CASE}

I now turn to the case when the agent is able to save and borrow in an account that the principal cannot monitor. As discussed above, this means that the agent's wealth $m_{t}$ evolves as in (3), and the principal can no longer guarantee that the agent consumes his payment. The principal tries to deter savings, and thus targets $\hat{m} \equiv 0$ and $\hat{c}=s$, but he must now structure the contract so that the agent has no incentive to save. As under hidden actions, I solve this problem by first deriving the agent's optimality conditions facing a given history-dependent contract. However unlike the hidden action case, I can no longer show that the agent's optimality conditions characterize the set of implementable contracts. Thus I solve for a candidate optimal contract using the necessary optimality conditions, then show ex-post that the contract is truly incentive compatible and therefore implementable. Werning (2001), Abraham and Pavoni (2008), and Farhi and Werning (2013) follow the same approach, and here I am able to verify incentive compatibility analytically. In general with hidden state variables, the contract must condition on an additional endogenous state 
variable, capturing the shadow value (in terms of the agent's marginal utility) of the hidden state. But in my the environment the additional variable is redundant.

\subsection{The agent's problem}

As under hidden actions, I first derive the agent's optimality conditions facing a given contract. Now the agent chooses consumption as well as effort, so his problem is to solve:

$$
\sup _{(\bar{e}, \bar{c}) \in \mathcal{A}} V(\bar{e}, \bar{c})
$$

subject to (7)-(8), given $\bar{s}$. Again, I apply stochastic maximum principle, and the additional state variable necessitates an additional adjoint. The Hamiltonian $\mathcal{H}$ for the agent's problem with states $\left(\Gamma_{t}, x_{t}\right)$ and adjoints $\left(q_{t}, \gamma_{t}\right)$ and $\left(p_{t}, Q_{t}\right)$ can be written as $\mathcal{H}=\Gamma H$ with:

$$
H(y, m, e, c, d, s, \gamma, p, Q)=u(c, e)+(\gamma+Q m)(r y+B e-s-d)+p(r m+s-c),
$$

where I use $x=\Gamma m$. Differentiating the Hamiltonian gives the evolution of the co-states, with $q_{t}$ again following (10), now with the terminal condition $q_{T}=v\left(s_{T}, m_{T}\right)$. Again $q_{t}$ is the promised utility and $\gamma_{t}$ is its sensitivity. The co-state variable $p_{t}$ with sensitivity $Q_{t}$ is associated with the (scaled) wealth $x_{t}$ and follows:

$$
d p_{t}=(\rho-r) p_{t} d t+Q_{t} \sigma d W_{t}^{\bar{e}}, \quad p_{T}=v_{m}\left(s_{T}, m_{T}\right) .
$$

This is derived by differentiating $\mathcal{H}$ with respect to $x$ and then changing measure. The stochastic maximum principle then gives necessary conditions for the agent's optimal choice.

Proposition 5.1. Let $\left(e^{*}, c^{*}, \Gamma^{*}, x^{*}\right)$ be an optimal control-state pair. Then there exist $\mathcal{F}_{t^{-}}$-adapted process $\left(q_{t}, \gamma_{t}\right)$ and $\left(p_{t}, Q_{t}\right)$ in $L^{2}$ that satisfy (10) and (19) with $(e, c)=\left(e^{*}, c^{*}\right)$. Moreover the optimal control $\left(e^{*}, c^{*}\right)$ satisfies for almost every $t \in[0, T]$ almost surely:

$$
H\left(y_{t}, m_{t}^{*}, e_{t}^{*}, c_{t}^{*}, d_{t}, s_{t}, \gamma_{t}, p_{t}, Q_{t}\right)=\max _{(e, c) \in A} H\left(y_{t}, m_{t}^{*}, e, c, d_{t}, s_{t}, \gamma_{t}, p_{t}, Q_{t}\right) .
$$

Suppose in addition that $A$ is convex, then an optimal control $\left(e^{*}, c^{*}\right)$ must satisfy for all $(e, c) \in A$, almost surely:

$$
\begin{aligned}
& H_{e}\left(y_{t}, m_{t}^{*}, e_{t}^{*}, c_{t}^{*}, d_{t}, s_{t}, \gamma_{t}, p_{t}, Q_{t}\right)\left(e-e_{t}^{*}\right) \leq 0 \\
& H_{c}\left(y_{t}, m_{t}^{*}, e_{t}^{*}, c_{t}^{*}, d_{t}, s_{t}, \gamma_{t}, p_{t}, Q_{t}\right)\left(c-c_{t}^{*}\right) \leq 0
\end{aligned}
$$

For interior optima, the first order conditions are:

$$
\begin{aligned}
(\gamma+Q m) B & =\lambda e \exp \left(-\lambda\left(c-e^{2} / 2\right)\right) \\
p & =\lambda \exp \left(-\lambda\left(c-e^{2} / 2\right)\right) .
\end{aligned}
$$

Thus $p_{t}=u_{c}\left(c_{t}, e_{t}\right)$, the agent's marginal utility of consumption. This is intuitive, as with a little additional wealth the agent could increase his current consumption marginally. The 
solution to (19) can be written:

$$
p_{t}=E\left[e^{(r-\rho)(T-t)} v_{m}\left(s_{T}, m_{T}\right) \mid \mathcal{F}_{t}\right]
$$

By the law of iterated expectations, this implies that a standard consumption Euler equation holds, as for $s>t$ :

$$
u_{c}\left(c_{t}, e_{t}\right)=E\left[e^{(r-\rho)(s-t)} u_{c}\left(c_{s}, e_{s}\right) \mid \mathcal{F}_{t}\right] .
$$

The agent's optimality conditions pin down the expected evolution of the marginal utility of consumption, while the contract influences the volatility of marginal utility $Q_{t}$.

\subsection{Necessary conditions for implementability}

In general, an implementable contract would need to condition on this marginal utility $p_{t}$ as well as the promised utility $q_{t}$ under the target policy $(\hat{e}, \hat{c})$ and $\hat{m} \equiv 0$. But in our setting, the additional co-state variable is redundant. This is a consequence of the proportionality of utility and marginal utility with exponential preferences. To see this, note that at the terminal date $T$ we have:

$$
p_{T}=v_{m}\left(s_{T}, 0\right)=V_{T}^{\prime}\left(s_{T}\right)=-\lambda r V_{T}\left(s_{T}\right)=-\lambda r q_{T},
$$

so $p_{T}$ is proportional to $q_{T}$. In addition, $p_{t}=u_{c}\left(c_{t}, e_{t}\right)=-\lambda u\left(c_{t}, e_{t}\right)$ so $(22)$ implies:

$$
u\left(\hat{c}_{t}, \hat{e}_{t}\right)=E\left[e^{(r-\rho)(T-t)} v\left(s_{T}, 0\right) \mid \mathcal{F}_{t}\right] .
$$

Therefore we can write (9) as:

$$
\begin{aligned}
q_{t} & =E\left[\int_{t}^{T} e^{-\rho(s-t)} u\left(\hat{c}_{s}, \hat{e}_{s}\right) d s+e^{-\rho(T-t)} v\left(s_{T}, 0\right) \mid \mathcal{F}_{t}\right] \\
& =E\left[r \int_{t}^{T} e^{-\rho(s-t)} E\left[e^{(r-\rho)(T-s)} v\left(s_{T}\right) \mid \mathcal{F}_{s}\right] d s+e^{-\rho(T-t)} v\left(s_{T}, 0\right) \mid \mathcal{F}_{t}\right] \\
& =E\left[\left(r \int_{t}^{T} e^{-\rho(s-t)+(r-\rho)(T-s)} d s+e^{-\rho(T-t)}\right) v\left(s_{T}, 0\right) \mid \mathcal{F}_{t}\right] \\
& =E\left[e^{(r-\rho)(T-t)} v\left(s_{T}, 0\right) \mid \mathcal{F}_{t}\right] \\
& =-\frac{1}{\lambda r} E\left[e^{(r-\rho)(T-t)} V_{T}^{\prime}\left(s_{T}\right) \mid \mathcal{F}_{t}\right]=-\frac{1}{\lambda r} p_{t} .
\end{aligned}
$$

Therefore $p_{t}=-\lambda r q_{t}$ for all $t \in[0, T]$, and the marginal utility state $p_{t}$ provides no additional information beyond promised utility $q_{t}$.

With $m=0$ and $p=-\lambda r q$ the agent's optimality conditions determine the following functions:

$$
\gamma(e, q)=\frac{-\lambda r e q}{B}, \quad c(e, q)=\frac{e^{2}}{2}-\frac{\log (-r q)}{\lambda}
$$


and in addition $u(c(e, q), e)=r q$. A contract is now locally incentive compatible if it is consistent with these conditions. As in the hidden action case, these ensure that if the agent were to have no wealth $\left(m_{t}=0\right)$ then the target controls would satisfy his optimality conditions. However to establish full incentive compatibility I also must rule out cases where the agent would accumulate a different amount of wealth and choose different actions. As discussed in Williams (2008), a sufficient condition for implementability is for the Hamiltonian to be concave in $(m, e, c)$. This is analogous to the condition which ensures the sufficiency of the maximum principle in Zhou (1996). Unfortunately, this concavity assumption is difficult to verify, and is too strong for the model in this paper. Instead I focus on necessary conditions for implementability, find a candidate contract optimal contract from this potentially larger set, and then establish that the contract is indeed incentive compatible. The necessary conditions are:

Proposition 5.2. An implementable contract $(s, \hat{e}, \hat{c}) \in \mathcal{S}$ in the hidden savings case with target wealth $\hat{m} \equiv 0$ satisfies (i) the participation constraint, (ii) promise-keeping, and (iii) is locally incentive compatible.

\subsection{The optimal contract}

Once again defining the value function $J(t, y, q)$, the principal's problem is to choose a contract that satisfies participation, promise-keeping, and incentive constraints. Relative to the hidden action case, consumption is now pinned down by the function $c(e, q)$, in addition to the volatility being determined by $\gamma(e, q)$. The principal's HJB equation for $0<t<T$ now becomes:

$$
\begin{gathered}
\rho J(t, y, q)-J_{t}(t, y, q)=\max _{d, e}\left\{-\exp (-\lambda d)+J_{y}(t, y, q)[r y+B e-c(e, q)-d]+\right. \\
\left.J_{q}(t, y, q)(\rho-r) q+\frac{1}{2} J_{y y}(t, y, q) \sigma^{2}+J_{y q}(t, y, q) \gamma(e, q) \sigma^{2}+\frac{1}{2} J_{q q}(t, y, q) \gamma(e, q)^{2} \sigma^{2}\right\}
\end{gathered}
$$

again with the terminal condition $J\left(T, y_{T}, q_{T}\right)=V_{T}\left(y_{T}-s_{T}\right)$ where $q_{T}=V_{T}\left(s_{T}\right)$. The first order conditions for $(d, e)$, again suppressing function arguments, are:

$$
\begin{aligned}
\lambda \exp (-\lambda d) & =J_{y} \\
J_{y}(B-e)-J_{y q} \sigma^{2} \frac{\lambda r q}{B}+J_{q q} \sigma^{2} \frac{\lambda^{2} r^{2} q^{2} e}{B^{2}} & =0 .
\end{aligned}
$$

The value function and the optimal policies have the same form as the previous cases, albeit with different constants. Once again the value function is of the form:

$$
J(t, y, q)=\frac{j_{2}(t)}{q} \exp (-\lambda r y)
$$


for some function $j_{2}(t)$. The optimal policies are:

$$
\begin{aligned}
e^{h s} & =\check{e}, \quad \gamma^{h s}(q)=\gamma(\check{e}, q)=-\frac{\lambda \check{e} r q}{B}, \\
c^{h s}(q) & =c(\check{e}, q)=\frac{(\check{e})^{2}}{2}-\frac{\log r}{\lambda}-\frac{\log (-q)}{\lambda}, \\
d^{h s}(t, y, q) & =-\frac{\log \left(j_{2}(t) r\right)}{\lambda}+\frac{\log (-q)}{\lambda}+r y,
\end{aligned}
$$

where $\check{e}$ is a constant. Given $e=\check{e}$, the policies $\gamma^{h s}$ and $c^{h s}$ are determined by the agent's optimality conditions. The function $j_{2}(t)$ satisfies an ODE of the same form as before:

$$
j_{2}^{\prime}(t)=j_{2}(t)\left[r \log j_{2}(t)-\delta\right]
$$

where:

$$
\delta=2(r-\rho)-r\left[B \lambda \check{e}-\lambda(\check{e})^{2} / 2+2 \log (r)\right]+\lambda^{2} r^{2} \sigma^{2}\left(1-\check{e} / B+\lambda(\check{e})^{2} r / B^{2}\right)
$$

and $j_{2}(T)=\eta^{2}$. The solution of this ODE is thus:

$$
j_{2}(t)=\exp (\delta / r+[2 \log (\eta)-\delta / r] \exp [-r(T-t)]) .
$$

In addition, recalling the optimal effort choice $e^{*}(k)$ from $(17)$ we have:

$$
\check{e}=e^{*}(r)=\frac{B^{3}+\sigma^{2} \lambda B r}{B^{2}+2 \sigma^{2} \lambda r}
$$

If $k=r$ then all of the results with hidden savings agree with the hidden action case. As discussed in more detail below, hidden savings limits the ability of the principal to provide intertemporal incentives. When the agent has access to un-monitored assets which yield the risk free return $r$, the principal cannot distort the allocation intertemporally to make the effective return $k \neq r$, as he could with hidden actions but observable wealth.

In the infinite horizon limit, the state variables evolve as follows:

$$
\begin{aligned}
& d y_{t}=\left[\frac{2(r-\rho)}{r \lambda}+\sigma^{2} \lambda r\left(\frac{1}{2}-\frac{\check{e}}{B}+\frac{(\check{e})^{2}}{B^{2}}\right)\right] d t+\sigma d W_{t} \\
& d q_{t}=(\rho-r) q_{t} d t-\frac{\sigma \lambda r \check{e}}{B} q_{t} d W_{t} .
\end{aligned}
$$

Thus output again follows an arithmetic Brownian motion with constant drift, while the utility process follows a geometric Brownian motion. Thus the expected growth rate of promised utility is the same under hidden savings as full information, and is greater than under hidden actions. This is a consequence of the inability of the principal to affect the agent's intertemporal incentives with hidden savings.

\subsection{Verifying incentive compatibility}


The optimal contract above was derived only using the necessary optimality conditions from the agent's problem, and so is potentially not incentive compatible. I now verify that the contract is indeed implementable by explicitly solving the agent's problem facing the contract.

Under the contract, the agent receives a history dependent payment $s_{t}=c^{h s}\left(q_{t}\right)$, which depends on promised utility $q_{t}$. For the agent, $q_{t}$ is simply part of the specification of the contract, and from his vantage point it evolves as:

$$
\begin{aligned}
d q_{t} & =(\rho-r) q_{t} d t-\frac{\sigma \lambda \check{e} r}{B} q_{t} d W_{t}^{\check{e}} \\
& =(\rho-r) q_{t} d t-\frac{\sigma \lambda \check{r} r}{B} q_{t}\left(\frac{d y_{t}-\left(r y_{t}+B \check{e}-c^{h s}\left(q_{t}\right)-d^{h s}\left(t, q_{t}, y_{t}\right)\right) d t}{\sigma}\right) \\
& =\left[\rho-r-\lambda \check{e} r\left(e_{t}-\check{e}\right)\right] q_{t} d t-\frac{\sigma \lambda \check{r} r}{B} q_{t} d W_{t},
\end{aligned}
$$

with $q_{T}=v\left(s_{T}, 0\right)$. Here I use the fact that $W_{t}^{\check{e}}$ is the driving Brownian motion under the optimal contract for the principal's information set. Shirking and setting $e_{t}<\check{e}$ thus increases the expected growth rate of promised utility, as the principal would interpret it as a negative shock. Note also that the terminal condition determines $s_{T}\left(q_{T}\right)$ as the inverse of the $V_{T}$ function.

Without loss of generality, I assume that the agent's initial wealth is $m_{0}=0$, as initial wealth is observable and can be taxed away by the principal. Wealth then evolves as:

$$
d m_{t}=\left(r m_{t}+c^{h s}\left(q_{t}\right)-c_{t}\right) d t
$$

Thus the agent's value function $V(t, q, m)$ solves the HJB equation:

$$
\begin{aligned}
\rho V(t, q, m)- & V_{t}(t, q, m)=\max _{c, e}\left\{-\exp \left(-\lambda\left(c-e^{2} / 2\right)\right)+V_{m}(t, q, m)\left[r m+c^{h s}(q)-c\right]\right. \\
& \left.+V_{q}(t, q, m) q[\rho-r-\lambda \check{e} r(e-\check{e})]+\frac{1}{2} V_{t, q q}(q, m) q^{2} \frac{\sigma^{2} \lambda^{2} \check{e}^{2} r^{2}}{B^{2}}\right\}
\end{aligned}
$$

with the terminal condition $V\left(T, q_{T}, m_{T}\right)=V_{T}\left(s_{T}\left(q_{T}\right)+m_{T}\right)=q_{T} \exp \left(-\lambda r m_{T}\right)$.

It is easy to verify that the agent's value function is given by $V(t, q, m)=q \exp (-\lambda r m)$ for all $t$. Substituting this into the HJB equation, and taking first order conditions for $(c, e)$ gives:

$$
\begin{aligned}
\lambda \exp \left(-\lambda\left(c-e^{2} / 2\right)\right) & =V_{m}=-\lambda r q \exp (-\lambda r m), \\
\lambda e \exp \left(-\lambda\left(c-e^{2} / 2\right)\right) & =-\lambda \grave{r} q V_{q}=-\lambda \check{r} r \exp (-\lambda r m) .
\end{aligned}
$$

Taking ratios of the two equations gives $e=\check{e}$, and thus the target effort level is implementable. Effort is independent of $m$ so the prospective "double deviations" of shirking and saving, which typically cause problems for incentive schemes with hidden savings, are not 
TABLE 1.

Comparison of the policies for the optimal contracts under full information, hidden actions, and hidden savings.

\begin{tabular}{|l|c|c|c|}
\hline & Full Information & Hidden Action & Hidden Saving \\
\hline Effort, $e$ & $B$ & $e^{*}(k)=\frac{B^{3} r+\sigma^{2} \lambda B r k}{B^{2} r+2 \sigma^{2} \lambda k^{2}}$ & $e^{*}(r)=\frac{B^{3}+\sigma^{2} \lambda B r}{B^{2}+2 \sigma^{2} \lambda r}$ \\
Consumption, $c$ & $\frac{B^{2}}{2}-\frac{\log r}{\lambda}-\frac{\log (-q)}{\lambda}$ & $\frac{e^{*}(k)^{2}}{2}-\frac{\log k}{\lambda}-\frac{\log (-q)}{\lambda}$ & $\frac{e^{*}(r)^{2}}{2}-\frac{\log r}{\lambda}-\frac{\log (-q)}{\lambda}$ \\
Dividend, $d$ & $-\frac{\log \left(j_{0}(t) r\right)}{\lambda}+\frac{\log (-q)}{\lambda}+r y$ & $-\frac{\log \left(j_{1}(t) r\right)}{\lambda}+\frac{\log (-q)}{\lambda}+r y$ & $-\frac{\log \left(j_{2}(t) r\right)}{\lambda}+\frac{\log (-q)}{\lambda}+r y$ \\
\hline
\end{tabular}

problematic here. The optimality condition for $c$ then gives:

$$
c=\frac{\check{e}^{2}}{2}-\frac{\log (-r q)}{\lambda}+r m=c^{h s}(q)+r m
$$

Substituting this into the wealth equation gives $d m_{t}=0$. If the agent begins with $m_{0}=0$, then he will remain at zero wealth, will consume the optimal amount under the contract $c=c^{h s}(q)$, and will attain the value $V(0, q, 0)=q$. Therefore the optimal contract is indeed implementable, even though we could not guarantee it ex-ante.

\section{IMPLICATIONS OF THE OPTIMAL CONTRACTS \\ 6.1. Comparing the Different Cases}

Table 1 summarizes the optimal contracts under full information, hidden actions, and hidden savings. As we've seen, the policy functions bear a strong resemblance to each other. In each case, effort is constant, consumption is linear in the log of the promised utility $q$, and the principal's dividend is linear in $\log (-q)$ and the current assets $y$. The consumption policies also depend negatively on the agent's effective rate of return on assets, which is $r$ with full information, $k$ with hidden actions, and $r$ with hidden savings. As we've already seen, the main effect of the hidden action case relative to full information is the reduction of effort. We've also seen that to first order there is no effect on consumption. This can be seen in the consumption policies, as effort falls but so does the effective return as $k<r$, leading to at least partially offsetting effects.

The main difference between the hidden saving and the hidden action cases is the effective rate of return on the agent's savings. If the return on the agent's saving were $k$ then the optimal policies in the hidden action and hidden savings cases would coincide. But access to hidden saving limits the ability of the principal to provide intertemporal incentives. If $k<r<4 k$ then effort is decreasing in $k$, and hence consumption is as well. ${ }^{18}$ Since for small shocks we've seen that $k$ is smaller than $r$ by a term proportional to the shock variance, this will certainly hold for small shocks. Moreover $c^{h a}$ is decreasing in $k$ if $e^{*}(k)$ is, as effort

\footnotetext{
${ }^{18}$ Simple calculations give

$$
\frac{d e^{*}(k)}{d k}=\frac{\sigma^{2} B^{3} r \lambda(r-4 k)-2 \sigma^{4} B r \lambda^{2} k}{\left(B^{2} r+2 \sigma^{2} \lambda k^{2}\right)^{2}},
$$
}

which is clearly negative if $r<4 k$. 

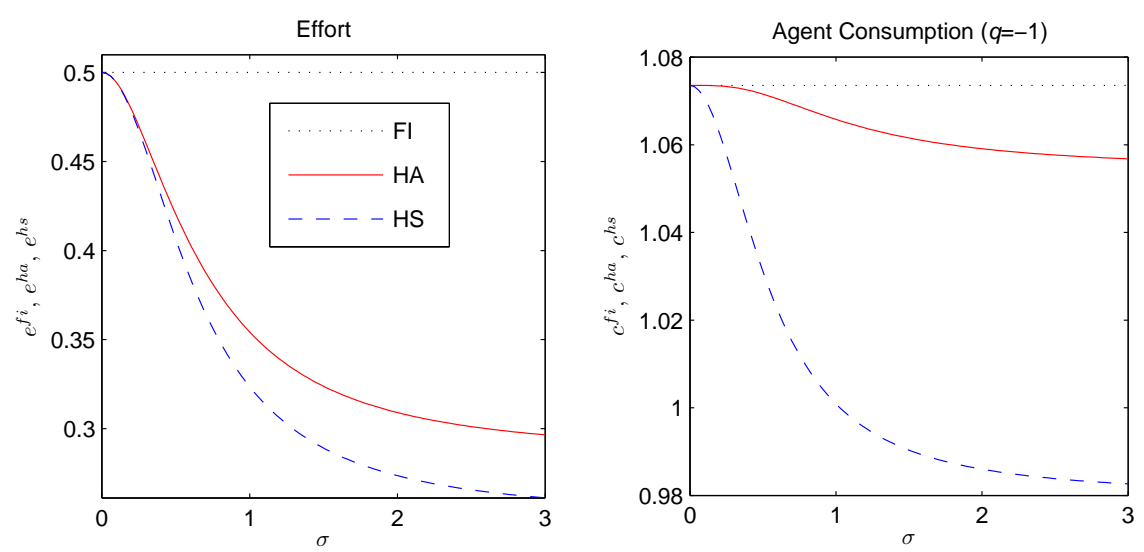

FIGURE 1. The agent's effort and consumption policies for different noise levels $\sigma$.

falls but the effective return rises. Thus at least for small enough $\sigma$, with hidden savings the agent puts forth less effort and consumes less than with hidden actions alone.

The analytic results are also borne out numerically. Figure 1 plots the functions for effort and consumption in the full information, hidden action, and hidden savings cases for a particular parameterization of the model. I set $r=0.15, B=0.5, \rho=0.1, \lambda=2$, and show the results for varying $\sigma$. In particular, the left panel plots effort versus $\sigma$, while the right panel plots consumption (evaluated at $q=-1$ ) versus $\sigma$. Clearly as $\sigma \rightarrow 0$ the cases all agree, as the information friction vanishes. Compared to full information, both effort and consumption fall under hidden actions. Moreover, for small $\sigma$ effort falls but consumption is relatively unaffected, as the approximations suggest. When the agent has access to hidden savings, consumption and effort fall further. In addition, these effects are all monotone in $\sigma$.

The policy functions also provide explicit expressions for the inefficiency "wedges," discussed by Kocherlakota (2004b) and others. These measure how the information frictions distort the consumption and labor allocations for incentive reasons. In particular, suppose that the agent were able borrow and lend at the same risk free rate $r$ as the principal. When the agent's saving is observable, the principal can tax it and drive its return down to $k$. Thus in the hidden action case, the contract introduces an intertemporal wedge $\tau^{K}$, a gap between the intertemporal marginal rate of substitution and the marginal return on assets. This is simply given by the tax rate which drives the after-tax rate of return down to $k$ :

$$
\tau^{K}(k)=1-\frac{k}{r}
$$

But if the principal cannot observe the agent's savings, he cannot tax it, so $\tau^{K}(r)=0$.

By varying the payment to the agent for incentive reasons, the optimal contract also induces a labor wedge $\tau^{L}$, a gap between the marginal productivity of effort and the marginal rate of substitution between effort and consumption. As the marginal product of effort is 

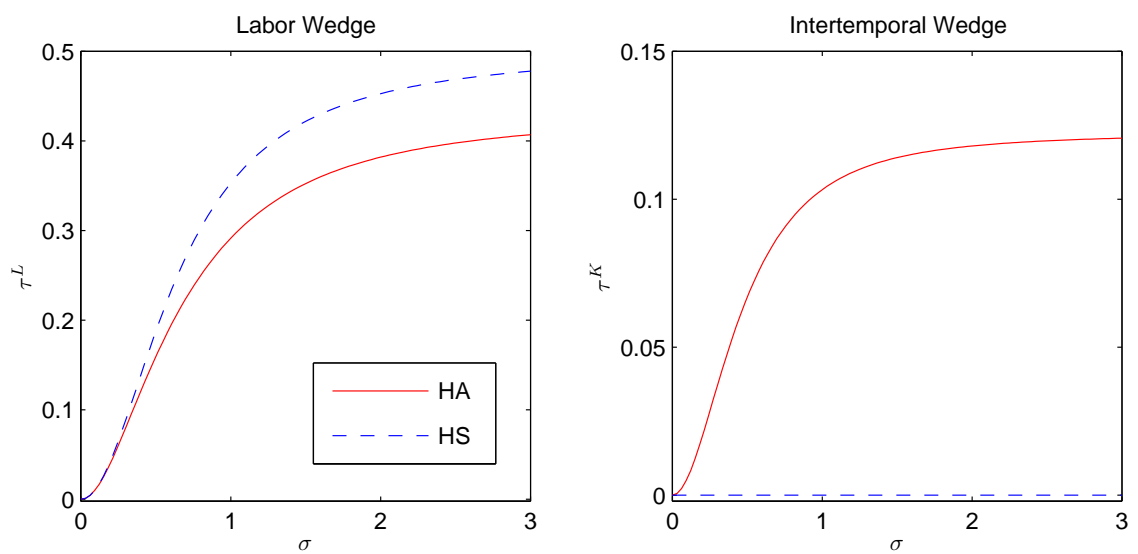

FIGURE 2. The labor and intertemporal wedges for different noise levels $\sigma$.

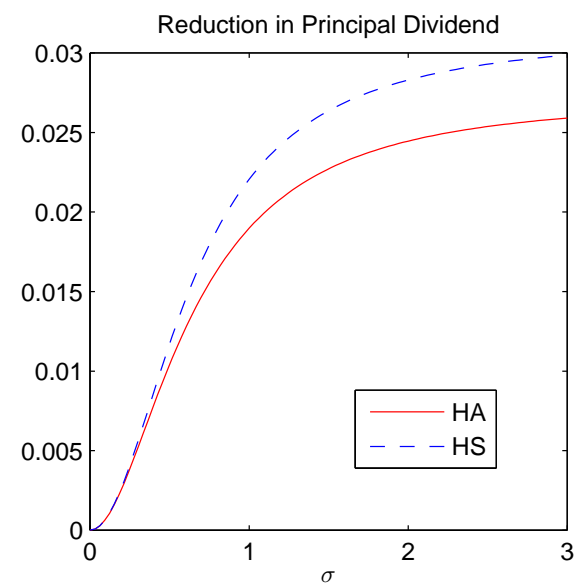

FIGURE 3. Reduction in the principal's dividend relative to the full information case for different noise levels

$B$ and the marginal rate of substitution is $e^{*}(k)$, the labor wedge is simply:

$$
\tau^{L}(k)=1-\frac{e^{*}(k)}{B} .
$$

Thus in the hidden saving case, the labor wedge increases relative to the hidden action case, as $\tau^{L}(r)>\tau^{L}(k)$ for $k<r<4 k$.

Both the intertemporal and labor wedges are constant, and are shown in Figure 2 for varying $\sigma$. The labor wedge is especially significant for this parameterization, as for $\sigma>1.5$ it is comparable to labor income tax rates of more than $30 \%$ under hidden actions and roughly $40 \%$ under hidden savings. The intertemporal wedge is smaller, being identically zero under hidden savings and flattening out for large $\sigma$ near an effective tax rate of $13 \%$ under hidden actions. 
Finally, reductions in the principal's consumption provide a measure of the cost of the information frictions. As Table 1 shows, the different informational assumptions affect $d$ only through the additive, time-dependent constants. Thus for each level of promised utility $q$ and assets $y$, at each date the principal's consumption differs by a constant amount depending on the information structure. Figure 3 plots the reduction (relative to the full information case) in the principal's dividend under hidden actions and hidden savings in the infinite horizon limit. The costs are relatively low, flattening out near 0.025 units of consumption under hidden actions and 0.03 units under hidden savings. As an example, with $y=0$ and $q=-1$ the dividend is exactly equal to the constant term, and these level reductions imply a 1.5-2\% fall in the principal's dividend. Of course with greater output or lower levels of promised utility, the proportional decline is much lower.

\subsection{Implementing the optimal allocations}

Thus far I have focused on a direct implementation of contracts, with the principal assigning consumption to the agent. I now show how the same outcomes can be achieved by giving the agent a share of the proceeds of the production, providing a constant flow payment (positive or negative depending on parameters), and then allowing the agent to invest in a risk-free asset (with a particular interest rate) and thus to choose his own consumption. The same implementation, with different parameters, works for all of the information structures. The agent's share of production is equal to one minus the labor wedge, his effective aftertax wage. In addition to providing direct compensation for putting forth effort, the equity stake makes the agent shares in the production risk. The risk-free rate the agent faces is one minus the intertemporal wedge, ensuring the appropriate dynamic incentives. Finally, the flow payment compensates the agent for risk and the difference between the effective interest rate and his rate of time preference.

In particular, suppose that we want to implement a contract which has shadow interest rate $\hat{r}$ and target effort $\hat{e}$. From our results above, the full information case is $\hat{e}=B, \hat{r}=r$, the hidden action case is $\hat{e}=e^{*}(k), \hat{r}=k$, and the hidden savings case is $\hat{e}=e^{*}(r), \hat{r}=r$. In addition, Table 1 shows that the consumption policies in each case can be written:

$$
c^{*}(q ; \hat{e}, \hat{r})=\frac{\hat{e}^{2}}{2}-\frac{\log (-\hat{r} q)}{\lambda}
$$

I now show how to implement the allocation from this contract. I focus on the infinite horizon limit for simplicity, but the same implementation works in the infinite horizon case, as only the principal's consumption is time-dependent. First, recall the proceeds of production $Y_{t}$ from (1) above, and now suppose that the agent receives a share $1-\tau^{L}=\frac{\hat{e}}{B}$ of this. In other words, the agent gets that equity stake in the firm. In addition, suppose that the agent has initial wealth $z_{0}=-\log \left(\hat{r} q_{0}\right) /(\lambda \hat{r})$. This includes any initial assets $m_{0}$ (which recall are observable even in the hidden state case) and a transfer from the principal. In addition to his production share, the agent receives a constant flow payment $\theta$ at each 
date, and he can borrow or lend at risk-free rate $\hat{r}$. Thus the agent's assets $z_{t}$ evolve as:

$$
\begin{aligned}
d z_{t} & =\left[\hat{r} z_{t}+\theta-c_{t}\right] d t+\left(1-\tau^{L}\right) d Y_{t} \\
& =\left[\hat{r} z_{t}+\hat{e} e_{t}+\theta-c_{t}\right] d t+\sigma \frac{\hat{e}}{B} d W_{t} .
\end{aligned}
$$

Thus the agent's HJB equation can be written:

$$
\rho V(z)=\max _{c, e}\left\{-\exp \left(-\lambda\left(c-e^{2} / 2\right)\right)+V^{\prime}(z)[\hat{r} z+\hat{e} e+\theta-c]+\frac{1}{2} V^{\prime \prime}(z) \sigma^{2} \frac{\hat{e}^{2}}{B^{2}}\right\}
$$

Calculations similar to those above show that the solution of this problem can be written $V\left(z_{t}\right)=-V_{a} \exp \left(-\lambda \hat{r} z_{t}\right)$, where $V_{a}$ is given by:

$$
V_{a}=\frac{1}{\hat{r}} \exp \left(\frac{\hat{r}-\rho}{\hat{r}}-\lambda\left(\frac{\hat{e}^{2}}{2}+\theta\right)+\frac{1}{2} \sigma^{2} \lambda^{2} \hat{r}^{2} \frac{\hat{e}^{2}}{B^{2}}\right),
$$

with the optimal policies:

$$
e=\hat{e}, \quad c=\frac{\hat{e}^{2}}{2}-\frac{\log \left(-\hat{r} V_{a}\right)}{\lambda}+\hat{r} z .
$$

Therefore the target effort is implementable and if we set the constant payment to:

$$
\theta=\frac{\hat{r}-\rho}{\lambda \hat{r}}-\frac{\hat{e}^{2}}{2}+\frac{1}{2} \sigma^{2} \lambda^{2} r^{2} \frac{\hat{e}^{2}}{B^{2}}
$$

then $V\left(z_{t}\right)=-\frac{1}{\hat{r}} \exp \left(-\lambda \hat{r} z_{t}\right)=q_{t}$, and:

$$
c=\frac{\hat{e}^{2}}{2}+\hat{r} z=\frac{\hat{e}^{2}}{2}-\frac{\log (-\hat{r} q)}{\lambda}=c^{*}(q ; \hat{e}, \hat{r}) .
$$

Thus this system of giving the agent a constant equity share of output, a constant flow payment, and a constant tax on his savings implements the optimal contract.

\section{CONCLUSION}

In this paper I have shown how to explicitly solve for optimal contracts under different information structures in a dynamic principal-agent model. By working in a continuous time setting, I was able to take advantage of powerful results in stochastic control. In addition by making exponential and linear functional form assumptions, I was able to derive explicit solutions and a simple implementation.

I showed that as in Holmstrom and Milgrom (1987) the optimal contract is linear in a fully dynamic setting with exponential utility. However now the payment is linear in an endogenous object, the logarithm of the agent's promised utility under the contract. Moreover, I showed that the main effect of hidden actions is to reduce effort, with a smaller effect 
on the agent's implicit rate of return under the contract, which in turn affects consumption. Introducing hidden savings eliminates this second distortion, and increases the effort distortion. I also showed how the optimal contracts could be implemented with the simple (and constant) instruments of an equity share, a tax on savings, and a flow payment.

\section{APPENDIX}

\section{A.1. DETAILS OF THE CHANGE OF MEASURE}

Here I provide technical detail associated with the change of measure in Section 2.4. I start by working with the induced distributions on the space of continuous functions, which I take to be the underlying probability space. Thus I let the sample space $\Omega$ be the space $C$, and let $W_{t}^{0}=\omega(t)$ be the family of coordinate functions, and $\mathcal{F}_{t}^{0}=\sigma\left\{W_{s}^{0} s \leq t\right\}$ the filtration generated by $W_{t}^{0}$. I let $P$ be the Wiener measure on $\left(\Omega, \mathcal{F}_{T}^{0}\right)$, and let $\mathcal{F}_{t}$ be the completion of $\mathcal{F}_{t}^{0}$ with the null sets of $\mathcal{F}_{T}^{0}$. This defines the basic (canonical) filtered probability space, on which is defined the Brownian motion $W_{t}^{0}$. Since $\sigma>0$ is constant, it clearly is continuous, invertible, and bounded. Thus it satisfies the regularity conditions required by Elliott (1982) to ensure that there exists a unique strong solution to the stochastic differential equation:

$$
d y_{t}=\sigma d W_{t}^{0},
$$

with $y_{0}$ given. This is the evolution of output under an effort policy $\bar{e}^{0}$ which makes the drift of output zero at each date. Different effort choices alter the evolution of output by changing the distribution over outcomes in $C$.

Now define the drift of output under a given contract $s(t, \bar{y})$ with the principal's consumption $d_{t}=d(t, \bar{y})$ as:

$$
f\left(t, \bar{y}, e_{t}\right)=r y_{t}-B e_{t}-s(t, \bar{y})-d(t, \bar{y})
$$

This drift is linear, and thus it satisfies the predictability, continuity, and linear growth conditions assumed by Elliott (1982). Then for $\bar{e} \in \mathcal{A}$ I define the family of $\mathcal{F}_{t}$-predictable processes:

$$
\Gamma_{t}(\bar{e})=\exp \left(\int_{0}^{t} \sigma^{-1} f\left(v, \bar{y}, e_{v}\right) d W_{v}^{0}-\frac{1}{2} \int_{0}^{t}\left|\bar{\sigma}^{-1} f\left(v, \bar{y}, e_{v}\right)\right|^{2} d v\right) .
$$

$\Gamma_{t}$ is an $\mathcal{F}_{t}$-martingale (as the form of $f$ ensures that Novikov's condition is satisfied) with $E\left[\Gamma_{T}(\bar{e})\right]=1$ for all $\bar{e} \in \mathcal{A}$. Thus by the Girsanov theorem, I can define a new measure $P_{\bar{e}}$ via:

$$
\frac{d P_{\bar{e}}}{d P}=\Gamma_{T}(\bar{e})
$$

and the process $W_{t}^{\bar{e}}$ defined by:

$$
W_{t}^{\bar{e}}=W_{t}^{0}-\int_{0}^{t} \bar{\sigma}^{-1} f\left(v, \bar{y}, e_{v}\right) d v
$$

is a Brownian motion under $P_{\bar{e}}$. Thus from (A.1), it's clear that the state follows the SDE:

$$
d y_{t}=f\left(t, \bar{y}, e_{t}\right) d t+\sigma d W_{t}^{\bar{e}} .
$$

Hence each effort choice $\bar{e}$ results in a different Brownian motion. $\Gamma_{t}$ defined above (suppressing $\bar{e}$ ) satisfies $\Gamma_{t}=$ $E\left[\Gamma_{T} \mid \mathcal{F}_{t}\right]$, and thus is the relative density process for the change of measure.

\section{A.2. PROOFS OF RESULTS}

Proof (Proposition 4.1 and 5.1). I condense the optimality conditions in the hidden action and hidden state cases into a single result, which follows by applying the results in Bismut (1973)-(1978). The result requires some basic regularity conditions on the fundamentals of the problem. Since $\sigma>0$ is constant, it clearly is continuous, invertible, and bounded. We have already defined $f$ as the drift of $y$ and now let $b=r m+s-c$ be the drift of $m$. Both $f$ and $b$ are linear, and thus are continuous and satisfy a linear growth condition. In addition, the period utility 
$u$ and terminal utility $v$ are continuously differentiable, as are the drift functions. Thus all the required conditions hold. I apply the maximum principle to the system with $(\Gamma, x)$ as state variables. I now illustrate the calculations leading to the Hamiltonian and the evolution of the adjoint equations (12) and (19). I first define the stacked system:

$$
X_{t}=\left[\begin{array}{c}
\Gamma_{t} \\
x_{t}
\end{array}\right], \Theta_{t}=\left[\begin{array}{c}
q_{t} \\
p_{t}
\end{array}\right], \quad \Lambda_{t}=\left[\begin{array}{c}
\gamma_{t} \\
Q_{t}
\end{array}\right] .
$$

Then note from (7)-(8) that $X_{t}$ satisfies (suppressing arguments):

$$
\begin{aligned}
d X_{t} & =\Gamma_{t}\left[\begin{array}{l}
0 \\
b
\end{array}\right] d t+\Gamma_{t}\left[\begin{array}{c}
\sigma^{-1} f \\
m_{t} \sigma^{-1} f
\end{array}\right] d W_{t}^{0} \\
& =M d t+\Sigma d W_{t}^{0}
\end{aligned}
$$

Thus, as in Bismut (1978), the Hamiltonian for the problem is:

$$
\mathcal{H}=\Theta M+\operatorname{tr}\left(\Lambda^{\prime} \Sigma\right)+\Gamma u=\Gamma H,
$$

where $H$ is from (18). As $\Gamma>0$, the optimality condition (20) is the same with $H$ or $\mathcal{H}$. The adjoint variables follow the BSDE:

$$
\begin{aligned}
d \Theta_{t} & =\left(\rho \Theta_{t}-\frac{\partial \tilde{H}}{\partial X}\right) d t+\Lambda_{t} d W_{t}^{0}, \\
\Theta_{T} & =\frac{\partial\left(\Gamma_{T} v\left(s_{T}, m_{T}\right)\right)}{\partial X_{T}} .
\end{aligned}
$$

By carrying out the differentiation and simplifying I arrive at (12) and (19).

Proof (Proposition 4.2). The result is an extension of Theorem 4.2 in Schattler and Sung (1993). The necessity of the conditions follow directly from my results above: if the contract is implementable then it clearly must satisfy the participation constraint, and by Proposition 4.1 it must satisfy promise-keeping and be locally incentive compatible.

To show the converse, I verify that $\hat{e}$ is an optimal control when the agent faces the contract $(s, \hat{e})$. Recall that the expected utility from following $\hat{e}$ is given by $V(\hat{e})=\hat{q}_{0}$, and any contract $(s, \hat{e})$ which satisfies promise-keeping (and so has a sensitivity variable $\hat{\gamma}$ ) has the following representation:

$$
v\left(y_{T}, 0\right)=q_{T}=\hat{q}_{0}-\int_{0}^{T} e^{-\rho t} u\left(s_{t}, \hat{e}_{t}\right) d t+\int_{0}^{T} \hat{\gamma}_{t} \sigma d W_{t}^{\hat{e}},
$$

which simply integrates (12) forward. Then for any $\bar{e} \in \mathcal{A}$ the following holds:

$$
\begin{aligned}
V(\bar{e})-V(\hat{e}) & =E_{\bar{e}}\left[\int_{0}^{T} e^{-\rho t}\left[u\left(s_{t}, e_{t}\right)-u\left(s_{t}, \hat{e}_{t}\right)\right] d t+\int_{0}^{T} \hat{\gamma}_{t} \sigma d W_{t}^{\hat{e}}\right] \\
& =E_{\bar{e}}\left[\int_{0}^{T} e^{-\rho t}\left[u\left(s_{t}, e_{t}\right)-u\left(s_{t}, \hat{e}_{t}\right)\right] d t+\int_{0}^{T} \hat{\gamma}_{t} \sigma d W_{t}^{\bar{e}}+\int_{0}^{T} \hat{\gamma}_{t} B\left(e_{t}-\hat{e}_{t}\right) d t\right] \\
& =E_{\bar{e}}\left[\int_{0}^{T}\left[H\left(y_{t}, e_{t}, s_{t}, d_{t}, \hat{\gamma}_{t}\right)-H\left(y_{t}, \hat{e}_{t}, s_{t}, d_{t}, \hat{\gamma}_{t}\right)\right] d t+\int_{0}^{T} \hat{\gamma}_{t} \sigma d W_{t}^{\bar{e}}\right] \\
& \leq E_{\bar{e}}\left[\int_{0}^{T} \hat{\gamma}_{t} \sigma d W_{t}^{\bar{e}}\right]=0 .
\end{aligned}
$$

Here the first equality uses (A.2), the second equality uses the definitions of the change of measure between $W^{\hat{e}}$ and $W^{\bar{e}}$, the third equality uses the definition of the Hamiltonian function $H$, the inequality follows from the local incentive constraint, and the final result uses the fact that the stochastic integral is a martingale due to the square integrability of the $\hat{\gamma}_{t}$ process. Thus since $\bar{e}$ was arbitrary, the agent can achieve at most the utility $V(\hat{e})$ which is greater than his reservation level by assumption. Thus $\hat{e}$ is an optimal control, and so the contract is implementable.

Proof (Proposition 5.2). The necessity of the conditions follows directly as in Proposition 4.2 above, as it is a consequence of Proposition 5.1.

\section{References}


Abraham, A. and N. Pavoni (2008). Efficient Allocations with Moral Hazard and Hidden Borrowing and Lending. Review of Economic Dynamics 11, 781-803.

Abreu, D., D. Pearce, and E. Stacchetti (1986). Optimal Cartel Equilibria with Imperfect Monitoring. Journal of Economic Theory 39, 251-269.

Abreu, D., D. Pearce, and E. Stacchetti (1990). Toward a Theory of Discounted Repeated Games with Imperfect Monitoring. Econometrica 58, 1041-1063.

Allen, F. (1985). Repeated Principal-Agent Relationships with Borrowing and Lending. Economics Letters 17, 27-31.

Bismut, J. M. (1973). Conjugate Convex Functions in Optimal Stochastic Control. Journal of Mathematical Analysis and Applications 44, 384-404.

Bismut, J. M. (1978). Duality Methods in the Control of Densities. SIAM Journal on Control and Optimization 16, 771-777.

Chari, V. V., P. J. Kehoe, and E. McGrattan (2007). Business Cycle Accounting. Econometrica 75, 781-836.

Cole, H. L. and N. Kocherlakota (2001). Efficient Allocations with Hidden Income and Hidden Storage. Review of Economic Studies 68, 523-542.

Cvitanić, J., X. Wan, and J. Zhang (2009). Continuous-Time Principal-Agent Problems with Hidden Action and Lump-Sum Payment. Applied Mathematics and Optimization 59, 99-146.

Cvitanic, J. and J. Zhang (2012). Contract Theory in Continuous-Time Models. New York: Springer-Verlag.

Doepke, M. and R. M. Townsend (2006). Dynamic Mechanism Design with Hidden Income and Hidden Storage. Journal of Economic Theory 126, 235-285.

Edmans, A., X. Gabaix, T. Sadzik, and Y. Sannikov (2012). Dynamic CEO Compensation. Journal of Finance 6r, 1603-1647.

Elliott, R. J. (1982). Stochastic Calculus and Applications. New York: Springer-Verlag.

Farhi, E. and I. Werning (2013). Insurance and Taxation over the Life Cycle. Review of Economic Studies 80, 596-635.

Fudenberg, D., B. Holmstrom, and P. Milgrom (1990). Short-Term Contracts and LongTerm Agency Relationships. Journal of Economic Theory 51, 1-31.

Garrett, D. and A. Pavan (2015). Dynamic Managerial Compensation: A Variational Approach. Journal of Economic Theory, forthcoming.

Holmstrom, B. and P. Milgrom (1987). Aggregation and Linearity in the Provision of Intertemporal Incentives. Econometrica 55, 303-328.

Jewitt, I. (1988). Justifying the First-Order Approach to Principal-Agent Problems. Econometrica 56, 1177-1190.

Kapicka, M. (2013). Efficient Allocations in Dynamic Private Information Economies with Persistent Shocks: A First Order Approach. Review of Economic Studies 80, 10271054. 
Kocherlakota, N. (2004a). Figuring Out The Impact of Hidden Savings on Optimal Unemployment Insurance. Review of Economic Dynamics 7, 541-554.

Kocherlakota, N. R. (2004b). Wedges and Taxes. American Economic Review (Papers and Proceedings) 94, 109-113.

Ma, J. and J. Yong (1999). Forward-Backward Stochastic Differential Equations and Their Applications, Volume 1702 of Lecture Notes in Mathematics. New York: SpringerVerlag.

Mirrlees, J. A. (1999). The Theory of Moral Hazard and Unobservable Behaviour: Part I. Review of Economic Studies 66, 3-21.

Mitchell, M. and Y. Zhang (2010). Unemployment Insurance with Hidden Savings. Journal of Economic Theory 145, 2078-2107.

Pavan, A., I. Segal, and J. Toikka (2014). Dynamic Mechanism Design: A Myersonian Approach. Econometrica 82, 601-653.

Rogerson, W. P. (1985). The First-Order Approach to Principal-Agent Problems. Econometrica 53, 1357-1367.

Sannikov, Y. (2008). A Continuous-Time Version of the Principal-Agent Problem. Review of Economic Studies 75, 957-984.

Sannikov, Y. (2013). Contracts: The Theory of Dynamic Principal-Agent Relationships and the Continuous-Time Approach. In D. Acemoglu, M. Arellano, and E. Dekel (Eds.), Advances in Economics and Econometrics: Tenth World Congress, Volume 1, pp. 89-124. New York: Cambridge University Press.

Schattler, H. and J. Sung (1993). The First-Order Approach to the Continuous-Time Principal-Agent Problem with Exponential Utility. Journal of Economic Theory 61, 331-371.

Spear, S. and S. Srivastrava (1987). On Repeated Moral Hazard with Discounting. Review of Economic Studies 53, 599-617.

Werning, I. (2001). Optimal Unemployment Insurance with Unobservable Saving. Working paper, MIT.

Williams, N. (2008). On Dynamic Principal-Agent Models in Continuous Time. Working paper, University of Wisconsin.

Williams, N. (2011). Persistent Private Information. Econometrica 79, 1233-1274.

Yong, J. and X. Y. Zhou (1999). Stochastic Controls. New York: Springer-Verlag.

Zhou, X. Y. (1996). Sufficient Conditions of Optimality for Stochastic Systems with Controllable Diffusions. IEEE Transactions on Automatic Control AC-41, 1176-1179. 
Figure
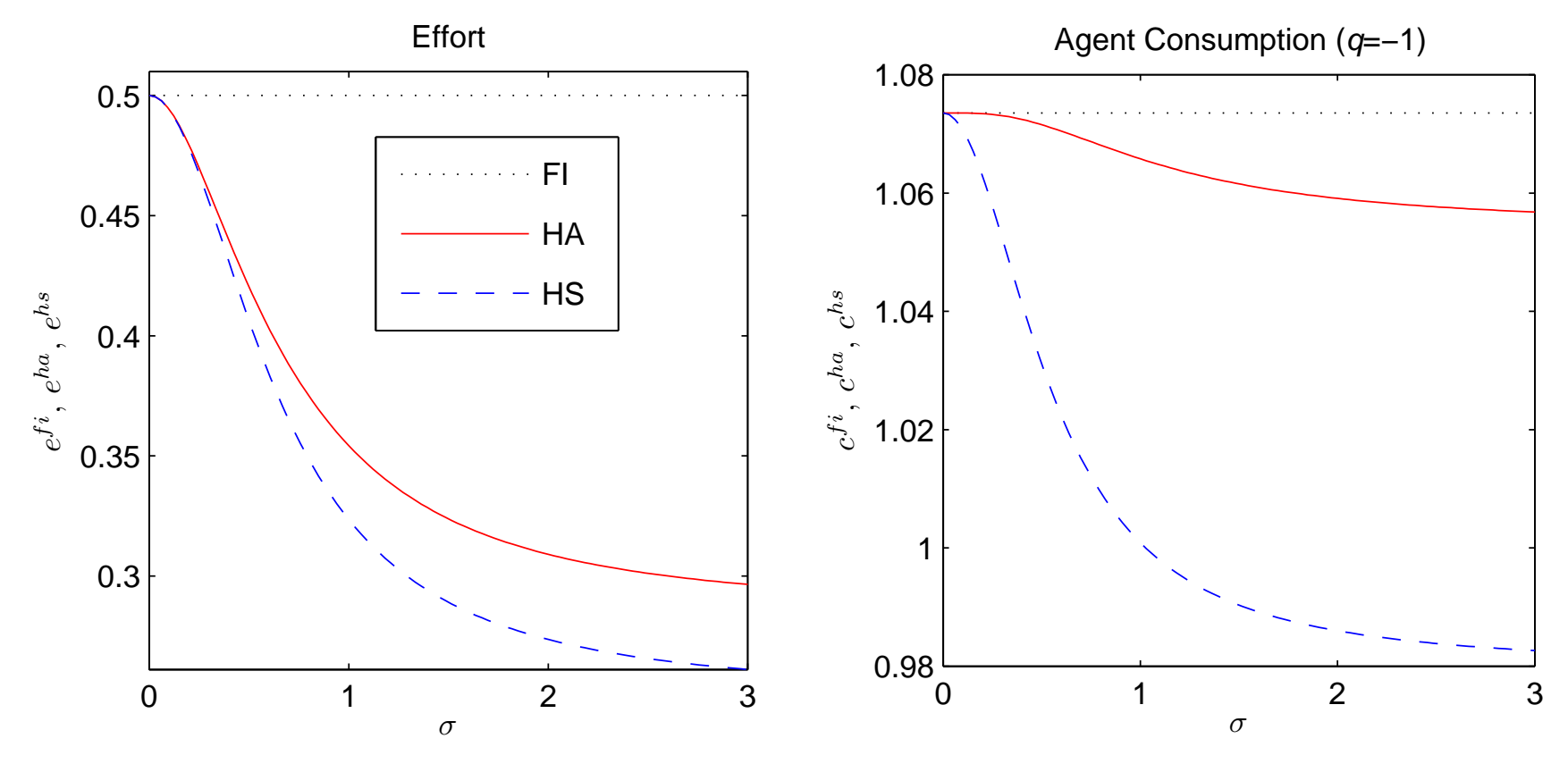

列


Figure
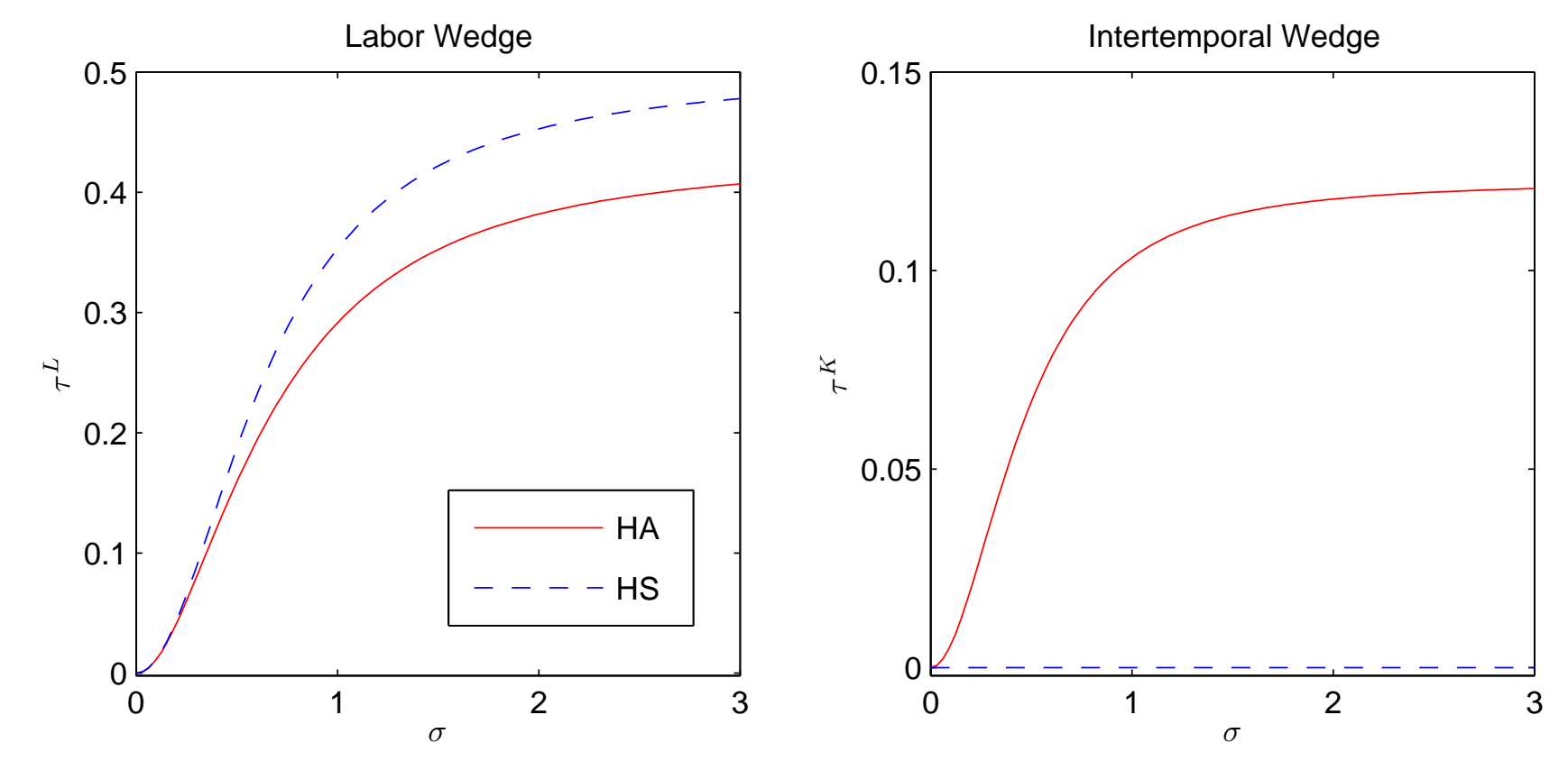

.

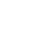




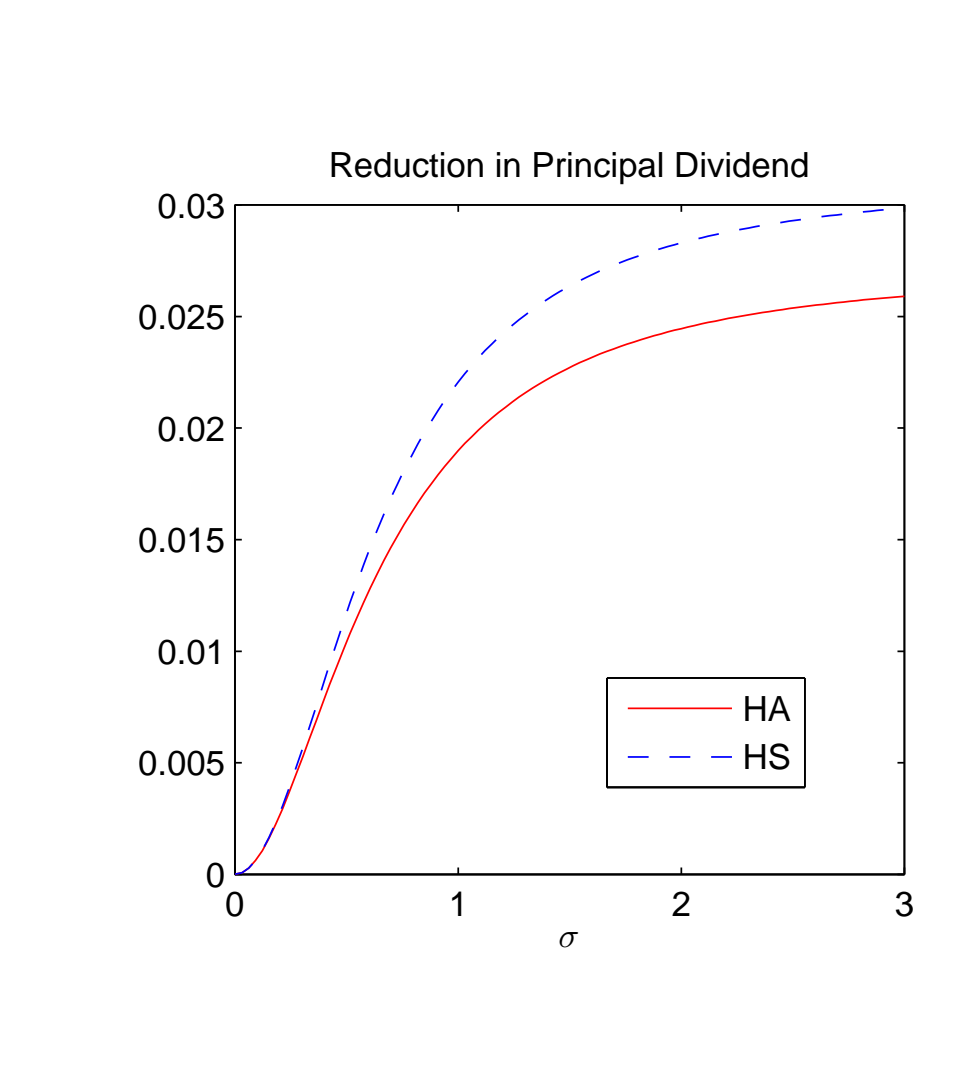

Figure

政

Figure

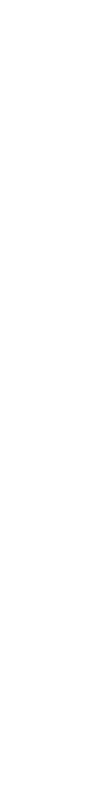

(n)

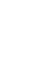

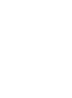

.
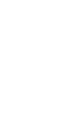